\title{
OPTIMAL JOB DESIGN IN THE PRESENCE OF IMPLICIT CONTRACTS*
}

\author{
ARIJIT MUKHERJEE ${ }^{\dagger}$ AND LUIS VASCONCELOS ${ }^{\ddagger}$
}

\begin{abstract}
We characterize the optimal job design in a multitasking environment when the firms rely on implicit incentive contracts (i.e., bonus payments). Two natural forms of job design are compared: (i) individual accountability, where each agent is assigned to a particular job and assumes full responsibility for its outcome; and (ii) team accountability, where a group of agents share responsibility for a job and are jointly accountable for its outcome. The key trade-off is that team accountability mitigates the multitasking problem but may weaken the implicit contracts. The optimal job design follows a cut-off rule: firms with high reputation concerns opt for team accountability, whereas firms with low reputation concerns opt for individual accountability. Team accountability is more likely the more acute the multitasking problem is. However, the cut-off rule need not hold if the firm combines implicit incentives with explicit pay-per-performance contracts.
\end{abstract}

\section{INTRODUCTION}

Firms frequently give work assignments to a group of employees (or "teams") and hold them jointly accountable for the outcome of the assignment. In fact, firms often adopt such a strategy even when it is technologically feasible to assign each worker to a particular job, and hold him solely accountable for his own performance (Bartol and Hagmann, 1992; Shaw and Schneier, 1995). Such a practice may seem counterintuitive because team performance can obscure individual contributions and blunt incentives. There is a vast literature on agency theory that studies the optimal incentive provisions in teams, but Corts (2007) is perhaps the first to explore how, in a multitasking environment, team accountability may arise endogenously when individual accountability is still a technologically feasible option. Corts argues that team accountability may optimally balance the trade-off between mitigating the multitasking problem and exposing the workers to a higher performance volatility.

This article contributes to the recent literature on endogenous job design by drawing out the implications for team accountability in the presence of implicit contracts, i.e., an informal promise by the firm to reward its worker(s) that is sustained through the threat of future retaliation of the worker(s) should the firm renege on its promise. ${ }^{1}$ Indeed, it is not hard to find real life examples where the firms offer implicit incentives in an environment where both multitasking and job design play a crucial role. Consider the mutual fund industry.

Date: December 31, 2010.

${ }^{*}$ We would like to thank Simon Anderson, Heski Bar-Isaac, Carl Davidson, Thomas Jeitschko, Pedro Pita Barros, Jon Levin, Helder Vasconcelos, Michael Waldman, Ján Zábojník and the seminar participants at Autoridade da Concorrência (Lisbon), Bates White LLC, McGill University, Michigan State University, the EARIE 2008 conference, 2009 SOLE Annual Meetings, and 2009 Labor Day Conference (University of Michigan) for their helpful comments. All errors that remain are ours.

${ }^{\dagger}$ Department of Economics, Michigan State University, 110 Marshall Adams Hall, East Lansing, MI 48824, USA. E-mail: arijit@msu.edu. URL: http://www.amukherjee.net

${ }^{\ddagger}$ Department of Economics, Universidade Nova de Lisboa, Campus de Campolide, 1099-032 Lisboa, Portugal. Email: l-vasconcelos@fe.unl.pt. URL: http://docentes.fe.unl.pt/ lipv064/.

${ }^{1}$ Such contracts are also referred to as "relational" contracts (Levin, 2003; Rayo, 2007) or "self-enforcing implicit" contracts (Bull, 1987). 
Managing a mutual fund may require a fund manager to exert effort to find new investment opportunities as well as to gather information about the risks associated with such investments. Even though both risks and returns significantly affect the overall profitability of the firm, a fund manager may be tempted to undertake high return investments without paying sufficient attention to the underlying risks. ${ }^{2}$ Traditionally each fund was individually managed by an assigned manager. However, there is a recent trend to move towards "comanaged" and/or "team managed" funds, where a group of employees are jointly responsible for the performance of a set of funds (see, e.g., Prather and Middleton, 2000; Chen, et al., 2004; and Massa, 2008). ${ }^{3}$ Implicit contracts, in the form of bonus payments, are ubiquitous in this industry.

As is the case with the mutual fund industry, there are two natural job designs: (i) individual accountability, where each agent is assigned to a particular job. An agent performs all of the tasks associated with the job, and the agent is solely responsible for the outcome of job he has been assigned to. (ii) Team accountability, where a group of agents share the responsibility for a job, each agent performs a subset of tasks associated with the job, and all agents are jointly accountable for the job outcome. ${ }^{4}$

In this article, we argue that while team accountability might mitigate the multitasking problem, it may make implicit contracts harder to sustain. Consider the following example that illustrates this trade-off: Suppose that the firm hires a worker (or a team of workers) to perform a job that involves two tasks - task 1 and 2. Let $e_{i}$ be the effort in task $i$, and the worker's cost of effort is $e_{i}^{2} / 2$. The workers' effort produces a value for the firm that is equal to $e_{1}+e_{2}$. That is, the firm cares about both tasks equally. Hence, the first-best effort levels (i.e., those that maximize the joint surplus $\left.e_{1}+e_{2}-\left(e_{1}^{2} / 2+e_{2}^{2} / 2\right)\right)$ are one unit of effort in each task $\left(e_{1}=e_{2}=1\right)$ and the resulting surplus level is 1 . The firm cannot observe the effort levels but can observe an imperfect measure of job performance, say, "success" or "failure," where $\operatorname{Pr}($ "success" $)=0.2 e_{1}+0.3 e_{2}$. The firm promises a bonus if the job is a "success." Note that even if the firm cares equally about both tasks, the worker has an incentive to exert more effort on task 2. Effort in task 2 has a higher marginal impact on the performance measure (or, probability of success) that determines the worker's bonus amount. Such an imperfection of the performance measure is the source of the multitasking problem.

\footnotetext{
${ }^{2}$ This might be particularly the case when the manager is primarily interested in increasing the fund's returns in the short run. The most visible signal of the manager's performance is perhaps the fund's returns over the last few quarters rather than the details of where the fund has been invested and what type of underlying risks it bears.

${ }^{3}$ In some cases, however, the difference between the two may not be one of job design but merely an issue of whether the firm is willing to share the credit (and blame) of the fund's performance with its employees (Massa, et al., 2008). The firm may also exploit potential economies of scope in production by assigning the same manager for both the tasks: finding investment and assessing risk. In this article we will abstract away from this issue of the production economies in order to stay focused on the multitasking problem. In fact, as we will discuss shortly, the benefit of individual accountability in our model stems from the economies of scope in incentive provision rather than the economies of scope in production.

${ }^{4}$ We use the terms "individual accountability" and "team accountability" in conformity with the existing literature on endogenous team formation (Corts, 2007). Note that this terminology indicates a classification of job design based on the associated incentive schemes. Alternatively, one can also think about this classification as one based on the type of specialization asked from the worker. Under "individual accountability," the worker specializes in a "job," whereas under "team accountability," the worker may specialize in a "task" (without any loss of generality, the model we use in Section 2 can be reformulated in this way). It is also important to note that the term "team" is used here in a slightly unconventional fashion. The term "team" often refers to a production process where no production is feasible unless a group of workers collaborate (mainly due to complementarities). In such settings, the firm assigns the job to a group of workers, and it is up to the workers to decide how they want to divide up the different tasks associated with the job. In contrast, we use the term "team" as a form of task assignment, where the firm instructs multiple workers to perform different parts of a job, even when it is technologically feasible for one worker to work on all parts.
} 
The firm decides whether to hire one worker to do both task 1 and 2 (i.e., adopt "individual accountability") or to hire two workers and assign each worker to one of the two tasks (i.e., adopt "team accountability"). Observe that if the firm resorts to individual accountability, to elicit the total effort (between the two tasks) under first-best-i.e., two units - the firm needs to offer $\$ 4$ in bonus: the worker chooses $e_{1}=0.8$ and $e_{2}=1.2$. Note that, while the aggregate effort level is two units, the worker overemphasizes task 2, creating an allocational inefficiency. The joint surplus produced is 0.96, less than the first-best level. Indeed, it is not possible to ensure equal effort in the two tasks because the firm must provide a single bonus payment to provide incentives for both tasks. Such a misalignment of effort across tasks reflects the underlying multitasking problem and leads to a loss of joint surplus.

Now suppose that the firm splits the job between the two workers: $A$ is in charge of task 1 and $B$ is in charge of task 2 . The firm can now resolve the multitasking problem by offering $\$ 5$ bonus to $A$ and $\$ 3.33$ bonus to $B$. Under these incentives, both $A$ and $B$ exert exactly one unit of effort, and, consequently, the first-best surplus is attained. The key issue to note is that, under team accountability, the firm can vary the power of incentives for the two workers, even if their pays are based on the same performance measure. Such flexibility in the incentive provisions alleviates the multitasking problem. However, observe that the total bonus requirement is now $\$ 8.33$; this is much higher than the bonus required to elicit two units of aggregate effort under individual accountability. Consequently, the firm's gains from reneging on its promise is also higher and it is harder for the firm to credibly commit to such a bonus pool. ${ }^{5}$ This is the basic trade-off with team accountability that we will explore in this article.

The benefit of team accountability in solving the multitasking problem has been discussed by several authors (Dewatriport, et al., 2000; Corts, 2007). The novel part of our analysis is to highlight the cost of team accountability in terms of weaker implicit incentives (or, equivalently, the need for larger bonus pool), and to draw out the implications of the tradeoff between mitigating the multitasking problem and weaker implicit incentives on the firm's job design decisions.

It is important to note that the necessity for a larger bonus pool under team accountability can be interpreted in terms of economies of scope in incentive provision. To see this point, consider the example discussed above. Under individual accountability, a single bonus payment (based on the job performance) offers incentives for efforts in both tasks associated with the job. The resulting economies of scope in incentive provision reduces the size of the bonus pool the firm needs to commit to. In contrast, under team accountability the job performance measure must be used twice to provide incentives for each of the two efforts separately (therefore two bonuses are required). Therefore the firm must commit to a larger bonus pool vis-a-vis the individual accountability case to provide the same incentive. One can also interpret the larger bonus requirement under teams as a cost of solving the underlying free-riding problem. The firm needs to offer separate bonus payments for each of the two tasks solely because a worker does not internalize the impact of his effort on the other agent's payoff (we will further elaborate on this issue in Section 3).

We formalize this trade-off between the mitigating multitasking problem and weakening of the implicit incentives in a stylized model similar to the example discussed above. We consider an environment where an infinitely lived principal (firm) hires two infinitely lived agents (workers). In each period, the two agents exert effort to perform two jobs. Both jobs involve two tasks each (i.e., there are four tasks in total). The effort exerted in each of the four tasks cannot be observed by the firm; this gives rise to a moral hazard problem.

\footnotetext{
${ }^{5}$ This effect is in sharp contrast with the credibility of promise discussed in the models of multilateral implicit contracts (e.g., Levin, 2002). In these models implicit contracts are easier to sustain when promises are multilateral rather than bilateral. We will further elaborate on this issue in section 3 .
} 
However, each of the two jobs yields an observable performance measure. The firm can offer incentives by tying an agent's compensation to the observed performance in the job(s) he has been assigned to. We further assume that the outcomes of both jobs are nonverifiable. Thus, the firm can only offer an implicit contract (i.e., bonus payment) to provide incentives for the two jobs.

The effort exerted in all four tasks has the same marginal benefit to the firm. But the effort spent on each of the two tasks related to any particular job affects the observed job performance differently. This gives rise to a multitasking problem. At the beginning of the game, the firm chooses between two alternative job designs: individual accountability and team accountability. In such a setting, we characterize the optimal job design as a function of the firm's discount factor $(\delta)$ that parameterizes its reputation concerns.

We show that the optimal job design follows a cut-off rule: team accountability is strictly optimal only if the firm's discount factor is sufficiently high. The intuition behind this result is simple but subtle. Recall that while team accountability allows the firm to overcome the multitasking problem, certeris paribus, it requires the firm to credibly commit to a larger bonus pool vis-a-vis individual accountability. If the firm's discount factor $(\delta)$ is sufficiently high, the threat of future punishment is significantly large for the firm, which, in turn, allows the firm to credibly promise to a larger bonus pool. Consequently, team accountability becomes optimal for firms with high $\delta$. However, for low $\delta$, the firm lacks credibility to offer a large bonus payments, and the firm is better off by resorting to individual accountability. Under individual accountability, even a small bonus payment gives sharper incentives because it elicits effort in all tasks associated with a particular job. In other words, individual accountability allows the firm to exploit the economies of scope in incentive provision. A single bonus payment provides some work incentives in all tasks, and the stronger incentives can outweigh the inefficiencies originating from the multitasking problem.

Given this basic intuition on how job design may interact with the implicit incentive provisions, we consider a more general setting where the workers' performances in a subset of jobs are indeed verifiable. In such an environment the firm may opt to provide incentives to its workers though a combination of explicit and implicit incentives. It may offer explicit payper-performance contracts to the agents assigned to the jobs where contractible performance measures are available and promise implicit contracts to the others. ${ }^{6}$

The scenario described above can be readily accommodated in our basic model. However, a new effect originates in the presence of the explicit contract: team accountability makes implicit incentives more fragile by enhancing the firm's punishment payoff. Following a breakdown of the implicit contract, the firm may only rely on the explicit contract for motivating the agents on the punishment path. Indeed, the firm's punishment payoff is simply the profit it earns from the jobs that are compensated by the explicit contracts. Because team accountability allows the firm to mitigate the multitasking problem, the explicit contracts are more efficient under team, and therefore, the firm's punishment payoff is higher under team accountability than under individual accountability. This makes implicit incentives even more fragile under team accountability. In other words, in the presence of explicit contracts, the punishment threats are weaker under team accountability, and this, in turn, increases the firm's temptation to cheat on its bonus promises.

This new effect invalidates the cut-off rule discussed above. Instead, we find that the optimal job design must be one of the following: (i) Only the firms with very high or very low $\delta$ opt for team accountability, while the firms with intermediate $\delta$ opt for individual accountability. (ii) Team accountability is optimal for all $\delta$. The former is the case when

\footnotetext{
${ }^{6}$ As we will discuss in section 4 , the commercial insurance industry is a typical example of such a multitasking environment.
} 
the extent of the multitasking problem is low; the latter is the case when the multitasking problem is severe.

The intuition behind this result is similar to the case of the cut-off result discussed above, except in the case of sufficiently low $\delta$. What drives the optimality of teams for low $\delta$ ? For $\delta$ sufficiently small, the firm has low reputation concerns, and hence, the implicit incentives are infeasible under both types of job designs. The firm's profit under team accountability is higher because the explicit incentive can elicit effort more efficiently (for the explicitly contracted job) under team setting by mitigating the multitasking problem. However, if the multitasking problem is sufficiently large, then even for a moderate $\delta$, the stronger implicit incentives under individual accountability need not be enough to compensate the associated multitasking problem. In this case, team accountability remains optimal for all values of $\delta$.

The main results of the article yield interesting comparative statics predictions by interlinking the key parameters to the firm's job design decision. For example, the cut-off result discussed above indicates that when only implicit incentives are available, team accountability becomes more likely as the firm's reputation concerns increase. Our results also imply that team accountability is more likely to be the optimal job design when the extent of the multitasking problem is severe. This finding, however, is quite intuitive, because the key benefit of team accountability that we highlight in this model is that of overcoming the multitasking problem. Thus, when the multitasking problem is severe, it favors team accountability over individual accountability.

Related literature: This article relates to two broad strands of literature on agency theoryincentives in team and implicit contracts - and highlights how team accountability may arise endogenously as the optimal job design in the presence of implicit contracts.

Both explicit and implicit contracts in teams are well studied in the literature (Holmström, 1982; Che and Yoo, 2003; Kvaloy and Olsen, 2006, Bar-Issac, 2007; Rayo, 2007). However, this literature generally assumes that team accountability arises exogenously and focuses solely on the incentive issues that may arise in teams. Two important exceptions are Itoh (1991) and Bar-Issac (2007), who argue that teamwork may indeed originate endogenously. But none of these authors considers the role of multitasking in team formation. ${ }^{7}$ Our article is perhaps more closely related to Corts (2007) who studies how team accountability may endogenously emerge as the optimal job design in a multitasking environment with explicit pay-per-performance contracts. Similar to our article, Corts highlights the benefit of team in overcoming the multitasking problem. However, he considers a different cost of team: teams make the agents' income more noisy, for which the risk-averse agents must be offered a higher risk premium. Corts shows that team accountability might be the optimal job design when the extent of the multitasking problem is high and/or the extent of risk aversion among the agents is low. Our article complements Corts' article by highlighting a different trade-off associated with the team accountability in the presence of implicit incentives. We abstract away from the risk aversion issue by assuming that the agents are risk neutral, but introduce a new friction in terms of implicit contracts. Drawing parallel to Corts, we find that team accountability emerges as an optimal job design when the extent of multitasking problem is large and/or the firm's concerns for future reputation (as represented by its discount factor) is high.

Another article that is closely related to ours is Levin (2002). Levin discusses the costs and benefits of multilateral contracting over bilateral contracting in employment relationships. In a multilateral contracting, similar to team accountability, the firm makes commitments to a

\footnotetext{
7Itoh's argument relies on the need to foster collaborations among employees (also see Ramakrishnan and Thakor, 1991). Bar-Issac shows how a team formed with the old and the young workers can restore the reputation concerns of the old workers, who otherwise have nothing more to prove to the outside labor market.
} 
large group of employees, whereas under bilateral contracting, similar to individual accountability, the firm makes commitments to individuals or small groups. There is no multitasking issue in Levin's model, and he highlights the trade-off that while multilateral contracting are difficult to adjust in response to exogenous shocks to the business environment, it facilitates implicit contracts. The latter effect is in sharp contrast with our article where team accountability hinders implicit incentive provisions. We will revisit this issue in Section 3.

Finally, our article is also related to two "sub-branches" of the agency theory literature: (i) multitasking (Holmström and Milgrom, 1991; Dewatripont, et al., 2000; Besanko, et al., 2005) and (ii) interaction between explicit and implicit incentives (Gibbons and Murphy, 1992; Baker, et al., 1994). In contrast with our article, the existing literature on multitasking primarily focuses on the explicit incentives. And the literature on the interaction between incentives primarily discusses the characteristics of the optimal contract (that emerges from such interplay between incentives) and is silent about its potential implications on job design in a multitasking environment. An important exception is Schöttner (2008). Schöttner investigates when to split three tasks between two agents, where both explicit and implicit contracts may be feasible. In her model, task-spliting can elicit the first-best effort level in one of the three tasks, but implicit contracts are sharper under "no-task-splitting." However, these benefits and costs of task-spliting originate from very different reasons (compared to our model), namely, specific restrictions on the agents' cost function and larger punishment threats when tasks are not split. Also, in contrast to our results, Schöttner finds that tasksplitting can be optimal only when implicit contracts are infeasible.

The article is organized as follows. Section 2 discusses the basic model and section 3 characterizes the optimal job design. The role of explicit contracts is discussed in section 4. Section 5 discusses the empirical implications of our main results and their robustness to alternative modeling assumptions. Finally, section 6 concludes. Unless mentioned otherwise, all proofs are in the appendix.

\section{The Basic Model}

Players. A long-run firm, $F$, hires two long-run agents, $A$ and $B$, to manage two funds. ${ }^{8}$

\section{Stage game}

In what follows we will describe the stage game that is played between the firm and the agents in each period $\tau \in\{1,2, \ldots\}$. The stage game is defined in terms of its four key ingredients: technology, job design, contracts, and the players' payoffs. We elaborate below on each of these four ingredients. ${ }^{9}$

Technology. The technology is modelled after the canonical task allocation model of Dewatripont et. al (2000). There are three central features to this class of models: (i) The firm only cares about the impact of the agents' effort to its bottom line (defined below as $V$ ); however, $V$ is not observed by the agents, (ii) the agents' compensations are based on a set of performance measures (defined below as $x$ ) that are observable but that are distinct from the firm's bottom line, and (iii) the marginal impact of the agents' efforts on $V$ and $x$ are different; this gives rise to a multitasking problem. ${ }^{10}$ The detailed description of the model is as follows.

\footnotetext{
${ }^{8}$ The reference to the mutual fund industry is only to maintain a parallel with the example discussed in the introduction. The model presented here does not purport to be a model of mutual funds but a general model of multitasking with implicit incentives.

${ }^{9}$ For the sake of clarity, while describing the stage game we will suppress the time suffix $\tau$ associated with each variable.

${ }^{10} \mathrm{~A}$ similar model is also adopted in Corts (2007).
} 
Suppose $F$ owns two funds, say 1 and 2, where in each period a fund performance can either be good or bad. Let $x_{i} \in\{0,1\}$ be the index for the fund's performance, where $x_{i}=1$ if the fund's performance is good and $x_{i}=0$ if it is bad $(i=1,2)$. We define managing a fund as a "job" (thus there are two jobs in total). Each job consists of two tasks: (i) finding investment opportunities that yield higher returns and (ii) assessing the underlying risks associated with such an investment opportunity. We denote tasks 1 and 2 as the tasks required for job 1 , and tasks 3 and 4 as those required for job 2. Task $j \in\{1,2,3,4\}$ requires an effort level of $e_{j} \in[0,1]$. Let the cost of effort in task $j$ be $c\left(e_{j}\right)=e_{j}^{2} / 2$. The tasks affect the value, $V$, that $F$ receives from the two funds, where

$$
V(\mathbf{e})=\phi \times\left(e_{1}+e_{2}+e_{3}+e_{4}\right)
$$

and $\phi>0$. The value $V$ is not observable to the agents. One may interpret $V$ as the impact of the agent's effort in various tasks to the bottom line of the firm's profitability. This value accrues directly to the firm and may not be clearly observed by the rank-and-file of a hierarchical organization. Furthermore, efforts are also unobservable, but each of the two jobs has performance measures, $x_{1}$ and $x_{2}$, that are observable. The efforts in each of the two jobs determine their performance as follows:

$$
\begin{aligned}
& \operatorname{Pr}\left(x_{1}=1 \mid \mathbf{e}\right)=e_{1}+\gamma e_{2}, \\
& \operatorname{Pr}\left(x_{2}=1 \mid \mathbf{e}\right)=e_{3}+\gamma e_{4},
\end{aligned}
$$

where $\gamma>1$. While $x_{1}$ and $x_{2}$ are observable, neither of them are verifiable. ${ }^{11}$ The parameter $\gamma$ measures the extent of multitasking problem. An agent who is compensated on the basis of $x_{1}$ (or $\left.x_{2}\right)$ has incentives to substitute away from $e_{1}$ (or $e_{3}$ ) and concentrate more on $e_{2}$ (or $e_{4}$ ), even though all tasks have the same marginal impact on the firm's value $(V)$. We assume the following parametric restriction to ensure that the probabilities in equation (1) are well defined (i.e., probabilities lie between 0 and 1 ) in any equilibrium of this game:

Assumption 1. $\phi<1 /(1+\gamma)$.

Assumption 1 requires $\phi$ to be small when $\gamma$ is large and vice versa. An important issue to note about this technology specification is that it rules out all interactions across efforts in different tasks. The costs $(c)$ and value $(V)$ are additively separable in efforts ruling out any substitutability or complementarity across tasks. The assumption of additive separability streamlines the model and improves the exposition of the key trade-off between multitasking and implicit incentives. This trade-off itself is not driven by this assumption, and we will discuss the impact of relaxing this assumption in section 5.

JoB DESIGN. Each agent is responsible for exactly two tasks. The type of allocation of tasks between the two agents is referred to as the job design. The firm chooses between two alternative job designs: individual accountability and team accountability. The agents have individual accountability when one is assigned to tasks 1 and 2 and the other is assigned to tasks 3 and 4. Thus, under individual accountability, one agent is responsible for job 1 (i.e., sole responsibility of managing fund 1), while the other one is responsible for job 2 (i.e., fund 2). Without loss of generality, we assume that under individual accountability, $A$ is responsible for job 1 (i.e., tasks 1 and 2), while $B$ is responsible for job 2 (i.e., tasks 3 and 4). In contrast, we assume that under team accountability, agent $A$ is assigned to the tasks 1

\footnotetext{
${ }^{11}$ We will relax this assumption in section 4.
} 
and 4 , while agent $B$ is assigned to tasks 2 and 3 . This is to say that, in a team, both agents have responsibility for both jobs.

It is important to note that in order to form teams one can also consider grouping tasks (1 and 3 ) and (2 and 4). This configuration has a natural interpretation that, under team accountability, each agent specializes in a task (say, " $\gamma$-task" or "non- $\gamma$-task") and performs this task for all jobs. However, we specify the grouping (task 1 and 4) and (task 2 and 3) to maintain a parallel with the case of individual accountability where each agent is responsible for exactly one " $\gamma$-task" (i.e., task 2 or 4). Such a specification is without loss of generality, because both configurations yield the same surplus to the firm (the total bonus requirement to implement a given effort profile e remains the same under the two configurations). ${ }^{12}$

Assumption 2. F decides on the job design at the beginning of the game, and its decision is irreversible.

Once a particular design is chosen, it is prohibitively costly to change it at a future date. This assumption simplifies the analysis of the firm's punishment payoff and allows us to draw out the implications of the key trade-off between the multitasking problem and sustenance of implicit contract more succinctly. ${ }^{13}$ With a slight abuse of notation, we add an auxiliary period, say $\tau=0$, at the beginning of the game to denote the stage when the firm makes its job design decision (this formulation will be useful below in defining the history and the strategies in the game).

Contracts. Because the performance in both jobs is nonverifiable, the firm can only offer an implicit contract promising (in each period) a bonus payment if the fund performance turns out to be good. So, under individual accountability (where agent $A$ is assigned to job 1 , and $B$ is assigned to job 2), a contract offered to agent $A$ (in each period) is a tuple $\left(W_{A}, \beta_{A}\right)$, where $W_{A}$ is a lump sum wage and $\beta_{A}$ an implicitly contracted bonus payment that is offered only if $x_{1}=1$. The contract offered to agent $B$ is of similar form. In contrast, in a team setting, a contract offered to agent $k \in\{A, B\}$ (in each period) is a tuple $\left(W_{k}, \beta_{k 1}, \beta_{k 2}\right)$, where $W_{k}$ is a lump sum wage and $\beta_{k i}$ is an implicitly contracted bonus payment that is offered only if $x_{i}=1$. $^{14}$

\footnotetext{
${ }^{12}$ Also note that a related feature of such team formation is that every agent is a member in multiple teams. This feature is, however, a modeling artifact and it is not essential for capturing the main trade-off we are interested in. One can use an alternative model similar to the numerical example discussed in the introduction where there is only one job involving two tasks. In this case, the question of job design boils down to the question of whether to hire one agent for both tasks or to hire two agents for each of the two tasks. We did not use such a model, because it has a potential drawback: the number of agents in the environment changes across the two forms of job design. Hence the firm's payoffs under team and individual accountability may not be readily comparable (especially when the reservation wages of the agents are strictly positive) leading to an additional complexity in our analysis.

${ }^{13}$ Note that this assumption does not affect the key trade-off between multitasking and sustenance of implicit contracts, and therefore, it does not affect the qualitative nature of our results. However, if this assumption is relaxed, on the punishment path, the firm will always choose the job design that leads to the highest punishment payoff. This effect will (weakly) increase the firm's punishment payoff under both individual and team accountability and enhance its temptation to cheat.

${ }^{14} \mathrm{An}$ important implication of such contract specification is that bonuses are assumed to be additively separable across jobs. While such a specification rules out certain interesting types of compensation schemes, e.g., relative performance evaluation, in some situations implementation of a "nonseparable" compensation scheme may not be feasible or it may involve additional costs to the firm (e.g., the job performance signals may not be public, it may create incentives for sabotage, it may encourage the workers to collude and shirk, etc.) However, as we will discuss in section 5, this assumption is not crucial for the qualitative nature of our findings but we maintain this assumption because it considerably improves the analytical tractability of our model.
} 
PAYOFfs. We assume that both the firm and the two agents are risk neutral. The payoff of the firm is simply the overall value that the firm receives from the two funds net of its expected wage payments. The payoff of an agent is the expected wages he receives net of the cost of effort. The exact expressions for the payoffs will depend on the job design. Under individual accountability, the firm's payoff in each period is

$$
\pi^{I}=V(\mathbf{e})-W_{A}-\beta_{A} \operatorname{Pr}\left(x_{1}=1 \mid \mathbf{e}\right)-W_{B}-\beta_{B} \operatorname{Pr}\left(x_{2}=1 \mid \mathbf{e}\right),
$$

and the payoffs of agent $A$ and $B$ are defined as

$$
\begin{aligned}
& u_{A}^{I}=W_{A}+\beta_{A} \operatorname{Pr}\left(x_{1}=1 \mid \mathbf{e}\right)-c\left(e_{1}\right)-c\left(e_{2}\right), \\
& u_{B}^{I}=W_{B}+\beta_{B} \operatorname{Pr}\left(x_{2}=1 \mid \mathbf{e}\right)-c\left(e_{3}\right)-c\left(e_{4}\right),
\end{aligned}
$$

respectively. In contrast, under team accountability, the firm's payoff is

$$
\pi^{T}=V(\mathbf{e})-\sum_{k \in\{A, B\}}\left[W_{k}+\beta_{k 1} \operatorname{Pr}\left(x_{1}=1 \mid \mathbf{e}\right)+\beta_{k 2} \operatorname{Pr}\left(x_{2}=1 \mid \mathbf{e}\right)\right]
$$

the payoff of the agent $A$ (who is assigned to the tasks 1 and 4 ) and $B$ (who is assigned to the tasks 2 and 3) are

$$
\begin{aligned}
& u_{A}^{T}=W_{A}+\beta_{A 1} \operatorname{Pr}\left(x_{1}=1 \mid \mathbf{e}\right)+\beta_{A 2} \operatorname{Pr}\left(x_{2}=1 \mid \mathbf{e}\right)-c\left(e_{1}\right)-c\left(e_{4}\right), \\
& u_{B}^{T}=W_{B}+\beta_{B 1} \operatorname{Pr}\left(x_{1}=1 \mid \mathbf{e}\right)+\beta_{B 2} \operatorname{Pr}\left(x_{2}=1 \mid \mathbf{e}\right)-c\left(e_{2}\right)-c\left(e_{3}\right)
\end{aligned}
$$

respectively. The outside option of both agents is assumed to be 0 .

Time Line. The sequence of events in a stage game (in an arbitrary period $\tau \geq 1$ ) is summarized as follows:

- At date $\tau .1$ (beginning of the period): $F$ offers contracts to the two agents $(W, \beta)$. Agents decide whether to accept or reject their contract. Game goes to period $\tau .2$ if at least one agent accepts his contract. Else the game ends.

- At date $\tau .2$ : Agents exert effort $\mathbf{e}$ in the tasks they have been assigned to.

- At date $\tau .3$ : Performance signals $x_{1}$ and $x_{2}$ in the two jobs are realized.

- At date $\tau .4$ (end of the period): $F$ pays the contracted lump sum wages $(W)$ and a bonus $\tilde{\beta}$ (which may be different from the implicitly promised bonus $\beta$ ).

\section{Repeated game}

The repeated game is simply the aforementioned stage game repeated in each period. Both the firm and the agents discount the future payoff at a common per period rate of $\delta \in[0,1)$.

History of the game. We denote the public history of play within period $\tau$ (or in the $\tau^{\text {th }}$ stage game) by $h_{\tau}$. For $\tau=0$, this history consists of $F$ 's choice of job design $d \in\{$ team accountability, individual accountability $\}$. Thus, $h_{0}=d$. For $\tau \geq 1, h_{\tau}$ is a tuple that reports: (i) the contract offered by $F$ to the two agents $\left(W_{A \tau}, W_{B \tau}\right)$; (ii) $A$ and $B$ 's decisions on whether to accept the contract, $z_{i \tau} \in\{$ accept, reject $\}, i \in\{A, B\}$; (iii) the outcomes in jobs 1 and $2, x_{1 \tau}$ and $x_{2 \tau}$; and (iv) $F$ 's actual bonus payments at the end of period $\tau, \tilde{\boldsymbol{\beta}}_{\tau}$. That is, $h_{\tau}=\left(\left(W_{A \tau}, W_{B \tau}\right),\left(z_{A \tau}, z_{B \tau}\right),\left(x_{1 \tau}, x_{2 \tau}\right), \tilde{\boldsymbol{\beta}}_{\tau}\right)$. We denote the public history of the game at the beginning of period $\tau$ by $h^{\tau}$ and the set of all possible public histories of the game at the beginning of period $\tau$ by $H^{\tau}$. For $\tau \geq 1, h^{\tau}=\left(h_{1}, h_{2}, . ., h_{\tau-1}\right)$. With a slight abuse of notation, we define $h^{0}=\varnothing$ and $H^{0}=\varnothing$. 
Strategies, Implicit CONTracts and equilibrium. We focus on pure strategy equilibria due to their analytical tractability. A strategy of $F, \sigma_{F}$, consists of the following decisions: (i) the decision made in period one on the job design $d$; (ii) the decisions made at the beginning of each period $\tau \geq 1$ on the contracts $\left(W_{A \tau}, W_{B \tau}\right)$, given the history $h^{\tau} \in H^{\tau}$; and (iii) the decisions made at the end of each period $\tau \geq 1$ on the actual bonus payments $\tilde{\boldsymbol{\beta}}_{\tau}$, given the history $h^{\tau} \in H^{\tau}$, contracts $\left(W_{A \tau}, W_{B \tau}\right)$ and outcomes $\left(x_{1 \tau}, x_{2 \tau}\right)$. A strategy of agent $i \in\{A, B\}, \sigma_{i}$, consists of the following decisions: (i) whether to accept the contract offered by $F$ at the beginning of each period $\tau \geq 1$, given the history $h^{\tau} \in H^{\tau}$; and (ii) the effort decisions in each period $\tau$ in which he accepts $F$ 's contract offer, given the history $h^{\tau} \in H^{\tau}$ and contract $\left(W_{A \tau}, W_{B \tau}\right)$.

For each period $\tau \geq 1$ and every history $h^{\tau} \in H^{\tau}$, an implicit contract between the firm and the two agents describes: (i) the compensation the firm should offer (and that should be paid) to both agents; (ii) whether the agents should accept or reject the firm's offer; and in the event of acceptance (iii) the agents' effort levels in the tasks they are assigned to. Because a part of the compensation (the bonuses) in this contract is only promised, the firm may have the incentive to renege on them once production occurs.

An implicit contract is self-enforcing if it constitutes a Perfect Public Equilibrium (PPE) in trigger strategies of the repeated game. ${ }^{15} \mathrm{~A}$ strategy profile $\left\langle\sigma_{F}, \sigma_{A}, \sigma_{B}\right\rangle$ constitutes a PPE in trigger strategies if: (i) given any public history, the strategy profile $\left\langle\sigma_{F}, \sigma_{A}, \sigma_{B}\right\rangle$ induces a Nash Equilibrium in the continuation game, and (ii) both agents revert back to playing their static best responses forever if $F$ reneges on its promise to any of the two agents. ${ }^{16}$

\section{Optimal Job Design}

The optimal job design is derived by comparing the firm's payoff under individual and team accountability when the associated incentive contracts are optimally chosen. Therefore, a characterization of the optimal job design requires a characterization of the optimal contracts. But before we do so, we briefly discuss the first-best solution to the firm's contracting problem.

The first-best solution serves as a benchmark for comparing the efficacy of each of the two job designs. The first-best solution is the one that maximizes the joint surplus between the firm and the two agents. That is, the first-best effort levels, $\mathbf{e}^{F B}$, solve $\max _{\mathbf{e}} V(\mathbf{e})-\sum c\left(e_{j}\right)$, or, equivalently, must satisfy the first-order conditions

$$
\phi=e_{j}^{F B} \forall j .
$$

Equation (2) suggests that the first-best effort in all four tasks should be the same and equal to $\phi$, the marginal benefit of effort to the firm.

We now focus on the optimal contracting under individual and team accountability and compare the efficiency of such contracts with the first-best outcome. The following lemma (à la Levin, 2003) simplifies the analysis by ensuring that without loss of generality, one can restrict attention to the class of stationary contracts; i.e., we can characterize the optimal contract in the repeated game as a tuple $\left(W_{A}, W_{B}, \beta_{A}, \beta_{B}\right)$ under individual accountability and as a tuple $\left(W_{A}, W_{B}, \beta_{A 1}, \beta_{B 1}, \beta_{A 2}, \beta_{B 2}\right)$ under team setting, where the optimal contract does not vary over time. (We omit the proof as it directly follows from the proof of Theorem 2 in Levin's article)

\footnotetext{
${ }^{15}$ See Fudenberg et. al (1994) for a detailed discussion of the PPE solution concept.

${ }^{16}$ Note that we have assumed that irrespective of the job design both agents trigger punishment if the firm cheats on at least one of the two agents. This specification, however, is not essential for our main results. For example, one can consider alternative specifications where under individual accountability agents do not observe each other's performance, and hence, the firm can cheat on one agent without drawing any punishment for the other.
} 
Lemma 1. (Levin, 2003) If an optimal contract exists, there exists a stationary contract that is also optimal.

Based on this observation we characterize below the optimal contracts under different job designs.

3.1. Individual Accountability. The optimal contract must satisfy three constraints: (i) Individual rationality $(I R)$, i.e., the contract must offer both agents rents at least as large as their outside options. (ii) Incentive compatibility $(I C)$, i.e., given the incentives, both agents choose their effort levels to maximize their expected payoffs. And finally, (iii) dynamic restriction $(D R)$, i.e., the firm's promise of bonus payments must be credible. We elaborate on each of these constraints below.

As the outside options of both agents are equal to 0 , given the contracts $\left(W_{A}, \beta_{A}\right)$ for agent $A$ and $\left(W_{B}, \beta_{B}\right)$ for agent $B$ and the prescribed effort levels e, the $(I R)$ constraints for agent $A$ and $B$ are:
$\left(I R_{A}^{I}\right)$
$W_{A}+\beta_{A} \operatorname{Pr}\left(x_{1}=1 \mid \mathbf{e}\right)-c\left(e_{1}\right)-c\left(e_{2}\right) \geq 0$,
$\left(I R_{B}^{I}\right)$
$W_{B}+\beta_{B} \operatorname{Pr}\left(x_{2}=1 \mid \mathbf{e}\right)-c\left(e_{3}\right)-c\left(e_{4}\right) \geq 0$.

Next, consider the $(I C)$ constraints. As we have discussed above, under individual accountability, agent $A$ is responsible for tasks 1 and 2 (i.e., job 1 ), and agent $B$ is responsible for tasks 3 and 4 (i.e., job 2). Given the implicitly contracted bonus payments $\beta_{A}$ (offered if $\left.x_{1}=1\right)$ and $\beta_{B}$ (offered if $x_{2}=1$ ), the optimization problems for the two agents are:

$$
\begin{aligned}
& \max _{e_{1}, e_{2}} W_{A}+\beta_{A} \operatorname{Pr}\left(x_{1}=1 \mid \mathbf{e}\right)-c\left(e_{1}\right)-c\left(e_{2}\right), \\
& \max _{e_{3}, e_{4}} W_{B}+\beta_{B} \operatorname{Pr}\left(x_{2}=1 \mid \mathbf{e}\right)-c\left(e_{3}\right)-c\left(e_{4}\right) \text {. }
\end{aligned}
$$

Thus, for any credible promise of bonus amounts, the agents' choice of effort levels must satisfy the following incentive compatibility conditions:
$\left(I C_{A}^{I}\right)$
$\beta_{A}=e_{1}=e_{2} / \gamma$
$\left(I C_{B}^{I}\right)$
$\beta_{B}=e_{3}=e_{4} / \gamma$

The $(I C)$ constraints above highlight the multitasking problem. Consider the case of job 1 that is assigned to agent $A$ (the case of job 2 is analogous). Given that both task 1 and task 2 are compensated based on the performance outcome in job 1, the effort levels exerted in these two tasks are linked by the relation $e_{1}=e_{2} / \gamma$. Because $\gamma>1$, for any value of $\beta_{A}$, agent $A$ will exert more effort in task 2 than in task 1 (similarly for $\beta_{B}$ ). And there cannot exist any values of $\beta_{A}$ and $\beta_{B}$ that can ensure the first-best allocation $e_{1}=e_{2}=\phi=e_{1}^{F B}=e_{2}^{F B}$.

Finally, consider the dynamic restriction $(D R)$ constraint. If the bonus promises are to be credible, the discounted value of the firm's payoff stream from offering such bonus payments (i.e., equilibrium payoff) must be greater than the bonus amount that the firm forfeits by reneging on its promise, plus the discounted value of the payoff stream the firm may earn if it reneges on its promise to one or both agents (i.e., punishment payoff). Now, by modeling specifications, if the firm reneges on its promise to at least one of the two agents, both agents revert back to their static best response, and do not exert any effort. Consequently, if the firm decides to renege on its promise, it is optimal to renege on both agents and its continuation 
payoff on the punishment path is zero. Hence, we must have the following constraint on $\beta_{A}$ and $\beta_{B}$ to ensure that reneging on bonus payments is not a profitable deviation for the firm:

$$
\frac{\delta}{1-\delta} \pi^{I} \geq \beta_{A}+\beta_{B}
$$

The optimal contracting problem for the firm can now be written as follows:

$$
\begin{gathered}
\max _{\mathbf{e}, W_{A} \cdot W_{B}, \beta_{A}, \beta_{B}} V(\mathbf{e})-W_{A}-W_{B}-\beta_{A} \operatorname{Pr}\left(x_{1}=1 \mid \mathbf{e}\right)-\beta_{B} \operatorname{Pr}\left(x_{2}=1 \mid \mathbf{e}\right) \\
\text { s.t. }\left(I R_{A}^{I}\right),\left(I R_{B}^{I}\right),\left(I C_{A}^{I}\right),\left(I C_{B}^{I}\right), \text { and }\left(D R^{I}\right) .
\end{gathered}
$$

This problem can be written as follows by eliminating $W_{A}, W_{B}$, and e by using the $(I R)$ and $(I C)$ constraints:

$$
\begin{array}{cc}
\max _{\beta_{A}, \beta_{B}} \pi^{I}\left(\beta_{A}, \beta_{B}\right) & =\phi(1+\gamma)\left(\beta_{A}+\beta_{B}\right)-\frac{1}{2}\left(1+\gamma^{2}\right)\left(\beta_{A}^{2}+\beta_{B}^{2}\right) \\
\text { s.t. } & \frac{\delta}{1-\delta} \pi^{I}\left(\beta_{A}, \beta_{B}\right) \geq \beta_{A}+\beta_{B}
\end{array}
$$

We can further simplify this problem by observing the fact that for any $\beta_{A}$ and $\beta_{B}, \pi^{I}\left(\beta_{A}, \beta_{B}\right)=$ $\pi^{I}\left(\beta_{A}, 0\right)+\pi^{I}\left(0, \beta_{B}\right)$ and $\pi^{I}\left(\beta_{A}, \beta_{B}\right)$ is concave in both $\beta_{A}$ and $\beta_{B}$. This observation implies that at the optimum, $\beta_{A}=\beta_{B} \equiv \beta$ (say), and the firm's optimal contracting problem boils down to:

$$
\mathcal{P}_{I}:\left\{\begin{array}{r}
\pi_{*}^{I} \equiv \max _{\beta} 2\left[\phi(1+\gamma) \beta-\frac{1}{2}\left(1+\gamma^{2}\right) \beta^{2}\right] \\
\text { s.t. } \quad \frac{\delta}{1-\delta}\left[\phi(1+\gamma) \beta-\frac{1}{2}\left(1+\gamma^{2}\right) \beta^{2}\right] \geq \beta . \quad\left(D R^{I}\right)
\end{array}\right.
$$

Let $r=(1-\delta) / \delta$. Lemma 2 below characterizes the optimal profit of the firm associated with the above contracting problem.

Lemma 2. The optimal profit under individual accountability is a continuous and monotonically decreasing function in $r$, given as follows:

$$
\pi_{*}^{I}(r)= \begin{cases}\phi^{2}(1+\gamma)^{2} /\left(1+\gamma^{2}\right) & \text { if } r \leq \frac{1}{2} \phi(1+\gamma) \\ 4\left(\phi(1+\gamma) r-r^{2}\right) /\left(1+\gamma^{2}\right) & \text { if } \frac{1}{2} \phi(1+\gamma)<r<\phi(1+\gamma) \\ 0 & \text { if } r \geq \phi(1+\gamma)\end{cases}
$$

For $r$ sufficiently large (i.e., $\delta$ sufficiently low), the optimal profit is simply equal to the punishment payoff of the firm, because no implicit incentives are feasible, even on the equilibrium path. As $r$ decreases (i.e., for larger values of $\delta$ ) the firm gains more credibility in promising implicit contracts. The resulting stronger implicit incentive induces greater effort and leads to an increase in the firm's profit until the maximal profit under individual accountability is achieved. ${ }^{17}$

\footnotetext{
${ }^{17}$ Observe that the maximal profit under individual accountability is less than the profit associated with the first-best, because the multitasking problem continues to prevail irrespective of how strong an implicit incentive the firm can credibly offer.
} 
While we present the formal proof of Lemma 2 in the Appendix, it is instructive to discuss the basic argument behind this lemma as it highlights the nature of the optimal incentive contract. Note that the solution to $\mathcal{P}_{I}$ is simply the highest $\beta$ feasible under $\left(D R^{I}\right)$ until the unconstrained maxima is reached. We can rewrite the $\left(D R^{I}\right)$ constraint as

$$
R^{I}(\beta):=\phi(1+\gamma) \beta-\frac{1}{2}\left(1+\gamma^{2}\right) \beta^{2} \geq r \beta
$$

The function $R^{I}(\beta)$ reflects the credibility of the firm when it promises a bonus of the amount $\beta$, and this function can be interpreted as the "reputation capital" (or per-period reputational capital) of the firm, given the bonus $\beta$. Thus, for any given $r$, the optimal $\beta$ is simply the largest $\beta$ that the firm can credibly promise until the value of $\beta$ that solves the unconstrained version of $\mathcal{P}_{I}$ becomes credible. The optimal bonus payment thus obtained, say $\beta_{*}^{I}(r)$, is given as:

$$
\beta_{*}^{I}(r)= \begin{cases}\phi(1+\gamma) /\left(1+\gamma^{2}\right) & \text { if } r \leq \frac{1}{2} \phi(1+\gamma) \\ (2 \phi(1+\gamma)-2 r) /\left(1+\gamma^{2}\right) & \text { if } \frac{1}{2} \phi(1+\gamma)<r<\phi(1+\gamma) \\ 0 & \text { if } r \geq \phi(1+\gamma)\end{cases}
$$

The optimal profit under individual accountability can now be obtained by plugging the value of $\beta_{*}^{I}(r)$ in $\pi^{I}$.

Having characterized the optimal contract and the associated profit of the firm under individual accountability, we next analyze the case of team accountability.

3.2. Team Accountability. Recall that without any loss of generality, we assumed that under team accountability, agent $A$ is responsible for tasks 1 and 4 , while agent $B$ is responsible for tasks 2 and 3. As in the case with individual accountability, we first discuss the $(I R)$, $(I C)$, and $(D R)$ constraints associated with the optimal contracting problem. The $(I R)$ constraints are analogous to the case of individual accountability and are given as follows:

$$
\begin{aligned}
& \left(I R_{A}^{T}\right) \quad W_{A}+\beta_{A 1} \operatorname{Pr}\left(x_{1}=1 \mid \mathbf{e}\right)+\beta_{A 2} \operatorname{Pr}\left(x_{2}=1 \mid \mathbf{e}\right)-c\left(e_{1}\right)-c\left(e_{4}\right) \geq 0, \\
& \left(I R_{B}^{T}\right) \quad W_{B}+\beta_{B 1} \operatorname{Pr}\left(x_{1}=1 \mid \mathbf{e}\right)+\beta_{B 2} \operatorname{Pr}\left(x_{2}=1 \mid \mathbf{e}\right)-c\left(e_{2}\right)-c\left(e_{3}\right) \geq 0 .
\end{aligned}
$$

However, the nature of the $(I C)$ and the $(D R)$ constraints is significantly different compared to the previous case. Consider the $(I C)$ constraints first. Given the bonus payments $\beta_{A 1}$ and $\beta_{B 1}$ (offered if $x_{1}=1$ ) and the bonus payments $\beta_{A 2}$ and $\beta_{B 2}$ (offered if $x_{2}=1$ ), the optimization problem for the two agents are:

$$
\begin{array}{ll}
\max _{e_{1}, e_{4}} & W_{A}+\beta_{A 1} \operatorname{Pr}\left(x_{1}=1 \mid \mathbf{e}\right)+\beta_{A 2} \operatorname{Pr}\left(x_{2}=1 \mid \mathbf{e}\right)-c\left(e_{1}\right)-c\left(e_{4}\right), \\
\max _{e_{2}, e_{3}} & W_{B}+\beta_{B 1} \operatorname{Pr}\left(x_{1}=1 \mid \mathbf{e}\right)+\beta_{B 2} \operatorname{Pr}\left(x_{2}=1 \mid \mathbf{e}\right)-c\left(e_{2}\right)-c\left(e_{3}\right) .
\end{array}
$$

Thus, the $(I C)$ constraints that the optimal contract must satisfy are:

$$
\begin{array}{ll}
\left(I C_{A}^{T}\right) & \beta_{A 1}=e_{1}, \beta_{A 2}=e_{4} / \gamma, \\
\left(I C_{B}^{T}\right) & \beta_{B 1}=e_{2} / \gamma, \beta_{B 2}=e_{3} .
\end{array}
$$


The $(I C)$ constraints above highlight both the benefit and the cost of team accountability. First, on the benefit side, it shows how team can mitigate the multitasking problem. Consider the case of job 1, where task 1 is performed by agent $A$ and task 2 is performed by agent $B$ (the case of job 2 is analogous). Under team accountability, the firm can vary the power of the incentives offered to agents $A$ and $B$ for each of the two tasks associated with job 1. Because two different agents are performing the two tasks, they can be paid differently for the same performance outcome in job 1 (i.e., $\beta_{A 1}$ need not be equal to $\beta_{B 1}$ ). Thus, the effort levels exerted in tasks 1 and 2 are no longer interlinked (as is the case with individual accountability), and for $\beta_{A 1}=\phi$ and $\beta_{B 1}=\phi / \gamma$, the first best allocation $\left(e_{1}=e_{2}=\phi\right)$ can be attained. Second, on the cost side, it shows why team accountability requires a higher bonus pool (compared to individual accountability) to extract the same amount of effort. Under individual accountability, the firm enjoys economies of scope in incentive provision. By promising a bonus payment of $\$ 1$ in a job, say job 1 , the firm can elicit effort in both tasks: one unit of effort in task 1 and $\gamma$ units of effort in task 2. But under team accountability, such economies of scope disappear. In order to elicit the same level of effort, the firm must pay $\$ 1$ to each of the two agents, i.e., the total amount of bonus that the firm must commit to is $\$ 2$. The need for a larger bonus pool can also be interpreted as a free-riding problem in teams - an agent chooses his effort levels to maximize his own payoff and ignores its impact on the other agent. ${ }^{18}$

Next, consider the dynamic restriction $(D R)$ constraint. Similar to the case of individual accountability, even under team accountability, both agents become aware of the firm's deviation (and hence, trigger punishment), even if the firm reneges its promise only with one of the two agents. Thus, if the firm decides to deviate, it is optimal to renege on both agents, and the punishment payoff of the firm would be 0 . Consequently, the relevant $(D R)$ constraint is

$$
\frac{\delta}{1-\delta} \pi^{T} \geq \beta_{A 1}+\beta_{B 1}+\beta_{A 2}+\beta_{B 2}
$$

The optimal contracting problem can now be formulated as follows:

$$
\begin{array}{r}
\max _{\mathbf{e}, W_{A} \cdot W_{B}, \beta_{A 1}, \beta_{A 2}, \beta_{B 1}, \beta_{B 2}} V(\mathbf{e})-W_{A}-W_{B}-\left(\beta_{A 1}+\beta_{B 1}\right) \operatorname{Pr}\left(x_{1}=1 \mid \mathbf{e}\right) \\
-\left(\beta_{A 2}+\beta_{B 2}\right) \operatorname{Pr}\left(x_{2}=1 \mid \mathbf{e}\right) \\
\text { s.t. }\left(I R_{A}^{T}\right),\left(I R_{B}^{T}\right),\left(I C_{A}^{T}\right),\left(I C_{B}^{T}\right), \text { and }\left(D R^{T}\right) .
\end{array}
$$

Using the $(I R)$ and $(I C)$ constraints to eliminate $W_{A}, W_{B}$ and $\mathbf{e}$, we can rewrite the problem as:

\footnotetext{
${ }^{18}$ As reflected by the agents' $(I C)$ constraints, we implicitly assume that the agents cannot collude between themselves. This is a natural assumption in our model, because we are primarily interested in exploring how the optimal job design is influenced by the reputation concerns of the firm (rather than the agents). Indeed, one can interpret the infinitely lived agents as a sequence of short-lived agents with perfect observability of the game's history (i.e., at the beginning of each period, two new agents are hired who leave the environment at the end of the period). This interpretation makes our model perhaps more realistic as most firms face a nontrivial rate of turnover in their workforce.
} 


$$
\begin{aligned}
& \max _{\beta_{A 1}, \beta_{B 1}, \beta_{A 2}, \beta_{B 2}} \pi^{T} \equiv \phi\left(\beta_{A 1}+\gamma \beta_{B 1}+\beta_{B 2}+\gamma \beta_{A 2}\right)-\frac{1}{2} \beta_{A 1}^{2}-\frac{1}{2} \gamma^{2} \beta_{B 1}^{2}-\frac{1}{2} \beta_{B 2}^{2}-\frac{1}{2} \gamma^{2} \beta_{A 2}^{2} \\
& \text { s.t. } \frac{\delta}{1-\delta} \pi^{T} \geq \beta_{A 1}+\beta_{B 1}+\beta_{A 2}+\beta_{B 2} . \quad\left(D R^{T}\right)
\end{aligned}
$$

This problem can further be simplified by virtue of the following observation: because, the agents are ex ante symmetric, and $\pi^{T}$ is concave and additively separable in $\beta \mathrm{s}$, at the optimum, we must have $\beta_{A 1}=\beta_{B 2}$ and $\beta_{B 1}=\beta_{A 2}$. Using this fact, we can rewrite the firm's problem as:

$$
\mathcal{P}_{T}:\left\{\begin{array}{c}
\pi_{*}^{T} \equiv \max _{\beta_{A 1}, \beta_{B 1}} 2\left[\phi\left(\beta_{A 1}+\gamma \beta_{B 1}\right)-\frac{1}{2}\left(\beta_{A 1}^{2}+\gamma^{2} \beta_{B 1}^{2}\right)\right] \\
\text { s.t. } \frac{\delta}{1-\delta}\left[\phi\left(\beta_{A 1}+\gamma \beta_{B 1}\right)-\frac{1}{2}\left(\beta_{A 1}^{2}+\gamma^{2} \beta_{B 1}^{2}\right)\right] \geq \beta_{A 1}+\beta_{B 1} . \quad\left(D R^{T}\right)
\end{array}\right.
$$

The above optimization program $\mathcal{P}_{T}$ is similar in spirit to its individual accountability counterpart $\mathcal{P}_{I}$. However three issues are important to note.

First, the $\left(D R^{T}\right)$ constraint offers a neat representation of the key trade-off associated with the team accountability. The $\left(D R^{T}\right)$ constraint indicates that, compared to individual accountability, under team accountability, the firm must commit to a larger bonus pool to elicit the same effort levels (because a separate bonus payment must be offered for each task). Consequently, team accountability makes implicit contracts difficult to sustain. However, given an aggregate bonus pool (i.e., $\beta_{A 1}+\beta_{B 1}$ ) the firm can vary the power of incentives offered for the two tasks (i.e., $\beta_{A 1}$ and $\beta_{B 1}$ do not have to be equal) and overcome the multitasking problem. ${ }^{19}$

Second, the $\left(D R^{T}\right)$ constraint is in sharp contrast with the dynamic restrictions discussed in the models of multilateral implicit contracts (Levin, 2002). In Levin's model, the dynamic restriction under multilateral contracts simply requires that the dynamic restriction under bilateral contracts must hold at the aggregate level (i.e., summed over all agents). Thus, the multilateral implicit contracts are easier to sustain. A similar effect is also discussed by Bernheim and Whinston (1990) in the context of multimarket contacts. In contrast, in the current setting, $\left(D R^{T}\right)$ is not an aggregate version of $\left(D R^{I}\right)$ (i.e., the $(D R)$ constraint under individual accountability as given in the program $\left.\mathcal{P}_{I}\right)$.

Third, the solution technique is slightly more complex than the method used in the case of individual accountability. This is due to the fact that now the firm needs to maximize with respect to two bonus amounts, $\beta_{A 1}$ and $\beta_{B 1}$, instead of one (as is the case in $\mathcal{P}_{I}$ ). We solve this problem in two steps. First, for a given value of total bonus payments $\beta=\beta_{A 1}+\beta_{B 1}$ we characterize the optimal individual bonus payments $\beta_{A 1}$ and $\beta_{B 1}$. Second, given the optimal $\beta_{A 1}$ and $\beta_{B 1}$ as a function of $\beta$, we find the optimal $\beta$ that the firm can sustain.

The following lemma characterizes the optimal profit of the firm under team accountability.

\footnotetext{
${ }^{19}$ Note that in our model, the free-riding problem in teams does not restrain the firm from achieving the first-best. As shown by Holmsrtom (1982), the free riding problem necessarily leads to a loss of efficiency in the absence of any "budget breaker" (i.e., under the constraint that the total wage bill for the team must be equal to the total output produced). Indeed, in our model, the firm is the residual claimant and works as the "budget breaker." Therefore, if the firm can offer sufficiently strong incentives, there is no loss of surplus due to free-riding and the first-best can be achieved (as we will show below).
} 
Lemma 3. The optimal profit under team accountability is a continuous and monotonically decreasing function in $r$ given as follows:

$$
\pi_{*}^{T}(r)= \begin{cases}2 \phi^{2} & \text { if } r \leq \frac{\phi \gamma}{\gamma+1} \\ 2 r K(r) / \gamma^{2} & \text { if } \frac{\phi \gamma}{\gamma+1}<r \leq \frac{1}{2} \phi(\gamma+1) \\ 4 r(\phi \gamma-r) / \gamma^{2} & \text { if } \frac{1}{2} \phi(\gamma+1)<r<\phi \gamma \\ 0 & \text { if } r \geq \phi \gamma\end{cases}
$$

where $K(r)=\phi \gamma(1+\gamma)-r\left(1+\gamma^{2}\right)+\left[\left(\phi \gamma(1+\gamma)-r\left(1+\gamma^{2}\right)\right)^{2}+\gamma^{2}(1-\gamma)^{2} \phi^{2}\right]^{1 / 2}$.

For $r$ sufficiently large (i.e., $\delta$ sufficiently small), the firm has little reputation concern, and hence, cannot credibly commit to any bonus payments. Consequently, it cannot induce any effort and makes zero profit. When $r$ decreases (i.e., $\delta$ increases), the firm can credibly commit to a positive but small amount of bonus payment. As discussed below in the context of equation (7), when the firm can only commit to a small amount of bonus payments, it offers bonuses only for the $\gamma$-tasks $\left(e_{2}\right.$ and $\left.e_{4}\right)$. As $r$ decreases even further, the firm finds it optimal to offer bonus for both tasks, and finally, for $r$ sufficiently small, the first-best effort level becomes feasible.

A discussion of the basic argument behind Lemma 3 is useful in understanding the nature of optimal contract under team accountability. We solve this problem in two steps. The first step is to solve an auxiliary problem of maximizing the firm's profit by choosing $\beta_{A 1}$ and $\beta_{B 1}$ when it can commit to a fixed amount of total bonus $\beta\left(=\beta_{A 1}+\beta_{B 1}\right)$. The following are the solutions to $\beta_{A 1}$ and $\beta_{B 1}$ in terms of the total bonus $\beta$ (for $\beta \leq \phi(\gamma+1) / \gamma$ ):

$$
\begin{array}{lll}
\beta_{A 1}^{T^{*}}=0, & \beta_{B 1}^{T^{*}}=\beta & \text { if } \beta<\phi \frac{\gamma-1}{\gamma^{2}} \\
\beta_{A 1}^{T^{*}}=\frac{\gamma^{2} \beta-\phi(\gamma-1)}{1+\gamma^{2}}, & \beta_{B 1}^{T^{*}}=\frac{\beta+\phi(\gamma-1)}{1+\gamma^{2}} & \text { if } \phi \frac{\gamma-1}{\gamma^{2}} \leq \beta<\phi \frac{\gamma+1}{\gamma} \\
\beta_{A 1}^{T^{*}}=\phi, & \beta_{B 1}^{T^{*}}=\frac{\phi}{\gamma} & \text { if } \quad \beta=\phi \frac{\gamma+1}{\gamma} .
\end{array}
$$

(Note that if $\beta>\phi(\gamma+1) / \gamma$, the solution is trivial, because the maximal profit is always attained by setting $\beta_{A 1}^{T^{*}}=\phi$ and $\beta_{B 1}^{T^{*}}=\phi / \gamma$.)

This solution has two important implications: First, it suggests that when the size of the available bonus pool $(\beta)$ is small, the firm should optimally give incentives only for the " $\gamma$-task" (i.e., task 2). However, as the amount of total available bonus increases, the firm starts offering bonuses for both tasks and eventually reaches the first best effort levels. The intuition behind this finding is as follows: for a dollar of bonus payment (offered if $x_{1}=1$ ), the marginal benefit of effort is higher if the effort is spent on the $\gamma$-task, i.e., task 2 (recall that $\gamma>1$ ). Thus, as long as the marginal cost of effort is moderate, a dollar promised for task 2 elicits more effort from the agent than a dollar promised for task 1 . When the firm cannot offer large bonus payments, the associated effort level is low, and so is the marginal cost of effort. Thus, in such a scenario, the firm is better off by offering the entire sum on task 2. But when a larger sum of bonus is offered, more effort is spent on task 2, and the marginal cost of effort associate with this task increases. Consequently, the marginal return of a dollar of bonus payment (in terms of the increment in effort induced) on task 2 decreases. In such a scenario, the firm finds it optimal to split the available bonus pool between the two tasks such that the marginal returns from bonus dollars offered for each task are equal. When the available bonus pool is significantly large, both $\beta_{A 1}$ and $\beta_{B 1}$ can be chosen appropriately so that the first-best effort levels become available. 
Second, equation (7) suggests that under the optimal contract, different members of the team receive different bonuses based on the same measure of performance (i.e., $\beta_{A 1}^{T^{*}} \neq \beta_{B 1}^{T^{*}}$ ). Moreover, the relative size of an agent's bonus is inversely related to his marginal contribution to the team's measured performance $(x)$. For example, as $\gamma$ increases, effort in task 2 has larger impact on the outcome of job 1. But agent $B$ 's (agent assigned to task 2) bonus payment, $\beta_{B 1}^{T^{*}}$, decreases in $\gamma^{20}$ This observation simply highlights the fact that team accountability allows the firm to align incentives with tasks within a job to mitigate the multitasking problem.

In the second step, we write the firm's profit function, and hence, the $\left(D R^{T}\right)$ constraint in terms of the total bonus pool $\beta$ using the solution as in equation (7). Now, similar to the case of individual accountability, the $\left(D R^{T}\right)$ constraint can be written as $R^{T}(\beta) \geq r \beta$ where $R^{T}(\beta)$ is the "reputation capital" of the firm when it promises a total bonus pool of $\beta$. Therefore, the firm's optimization problem boils down to the problem of finding the largest value of $\beta$ subject to the $(D R)$ constraint: $R^{T}(\beta) \geq r \beta{ }^{21}$ The optimal $\beta$ thus obtained is given as follows:

$$
\beta_{*}^{T}(r)=\left\{\begin{array}{ll}
\frac{1}{\gamma} \phi(\gamma+1) & \text { if } r \leq \frac{\phi \gamma}{\gamma+1} \\
\frac{1}{\gamma^{2}} K(r) & \text { if } \frac{\phi \gamma}{\gamma+1}<r \leq \frac{1}{2} \phi(\gamma+1) \\
\frac{2}{\gamma^{2}}(\phi \gamma-r) & \text { if } \frac{1}{2} \phi(\gamma+1)<r<\phi \gamma \\
0 & \text { if } r \geq \phi \gamma
\end{array},\right.
$$

where the function $K(r)$ is as defined in Lemma 3. When $\delta$ is very low (i.e., $r$ is very high) the firm does not have enough credibility to promise any bonus payment. As $\delta$ increases (i.e., $r$ decreases) the firm gradually gains credibility in committing to an increasing amount of bonus payment. When $\delta$ is sufficiently large (i.e., $r$ is sufficiently low) the firm has enough credibility to promise a large bonus pool that can implement the first-best effort levels. The optimal profit under team accountability can now be obtained by plugging the value of $\beta_{k i}^{T^{*}}$ s in $\pi_{T}$.

Equipped with the complete characterization of the firm's profit under team accountability and individual accountability, we can now address the issue of optimal job design.

3.3. Optimal job design. The optimal job design for a given value of $r$ (and hence, for a given $\delta$ ) is the one that yields the highest profit to the firm. A comparison between the optimal profits under individual and team accountability leads to the following characterization of the optimal job design.

Proposition 1. There exists a value of $r$, say $r^{*}$, such that team accountability is strictly optimal if and only if $r<r^{*}$.

\footnotetext{
${ }^{20}$ This finding is similar in spirit with a well-established result in incentive theory that the power of incentive may inversely vary with the sensitivity of the observed performance measure with respect to effort (see Banker and Datar, 1989). Indeed, the workers' incentive contracts often intentionally ignore certain observed performance measures so as to avoid the potential multitasking problem. For example, employment contracts for school teachers usually do not specify that teachers' pay be conditioned on the standardized test scores of their students, even though such scores are easily observed by both the employer and the employees.

${ }^{21}$ Of course, this is the case only until the unconstrained argmax value of $\beta$ is obtained.
} 
This proposition suggests that the optimal job design follows a cut-off rule: team accountability is optimal only for the firms with sufficiently high reputation concerns, i.e., sufficiently high $\delta$ (or, equivalently, sufficiently low $r$ ). And individual accountability is optimal otherwise. The key idea behind Proposition 1 is shown in figure 1 below. Figure 1 depicts an illustrative example of the optimal payoff from team $\left(\pi_{*}^{T}\right)$ and individual accountability $\left(\pi_{*}^{I}\right)$ as a function of the firm's discount rate represented by $r$. The optimal payoff functions intersect each other at only one point, $r^{*}$, where the payoff from team lies above the payoff from individual accountability for all $r<r^{*}$.

The intuition behind this result is simple. Recall that while team accountability allows the firm to overcome the multitasking problem, it also requires the firm to credibly commit to a larger bonus pool in order to elicit effort in all tasks. If the firm's discount factor $(\delta)$ is sufficiently high, the threat of future punishment is significantly large for the firm, which, in turn, allows the firm to credibly promise a high level of bonus payments. Thus, team accountability becomes optimal. However, for low $\delta$, the firm may not have the credibility to offer high bonus payments. In such a setting, the firm might be better off by resorting to individual accountability. Individual accountability offers economies of scope in incentive provisions where a single performance bonus payment based on the job outcome can simultaneously provide incentives for all tasks associated with the job. The sharper incentives under individual accountability may outweigh the inefficiencies that originate from the multitasking problem.

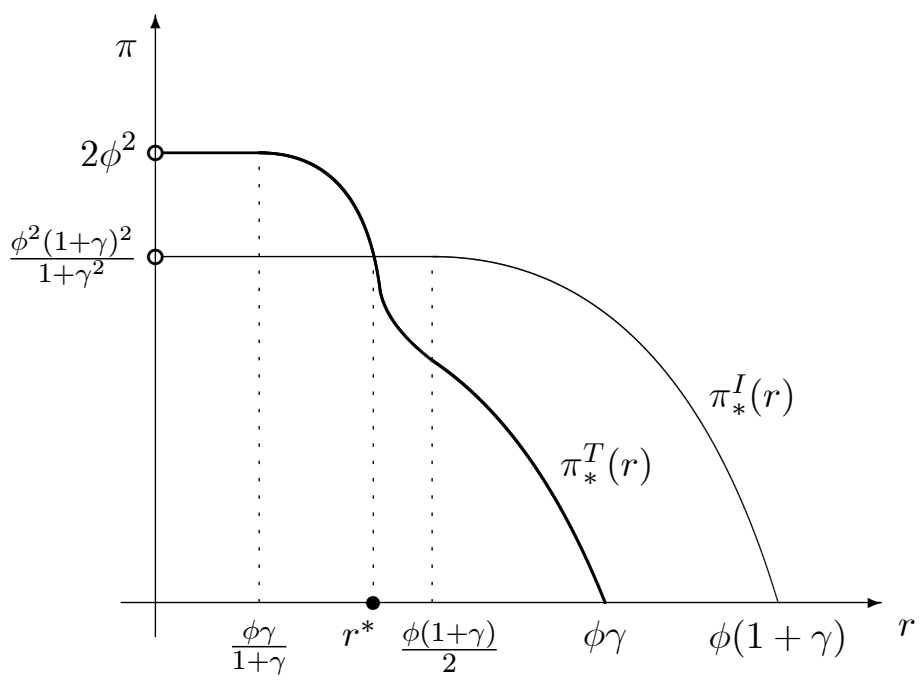

Figure 1. The maximal profits under team and individual accountability (team is optimal for $r<r^{*}$ )

Proposition 2. The threshold $r^{*}$ is increasing in $\gamma$.

The proposition above suggests that as the multitasking problem becomes more severe (i.e., as $\gamma$ increases), team accountability is more likely to be the optimal job design . This finding is quite intuitive, because the key benefit of team accountability is that it mitigates the multitasking problem. Thus, the more acute is the multitasking problem, the more likely it is that the firm will opt for teams. 


\section{INTERACTION BETWEEN EXPLICIT AND IMPLICIT INCENTIVES}

The previous section is instructive in drawing out the key trade-off associated with team accountability. But it does so under a simple framework where implicit contracts are the only form of incentives available. However, in many real life scenarios, firms augment implicitly contracted bonus incentives with explicit pay-per-performance contracts. A typical example of such an environment is the commercial insurance industry. The insurers rely on the agents (brokers) to perform two key jobs: (i) search for (commercial) clients who are willing to buy insurance coverage (i.e., search job) and (ii) elicit information about the clients' risk and coverage requirement to ensure that the insurer's offered coverage matches the clients' needs (i.e., match job). ${ }^{22}$ The performance of an agent on the search job has a contractible measure - the amount of business he brings to the insurer - and the agent is compensated through an explicitly contracted commission rate. The performance in the match job is, however, relatively hard to verify. Often, the agents are compensated for the match job through an implicitly promised bonus payment, or "contingency fee." 23 Both search and match jobs may involve multitasking problems. Effective search may require active solicitation of new business from existing clients as well as advertising the insurer's products to a broader clientele. Similarly, effective matching may require the agent to not only advise the client on the appropriate coverage but also to elicit accurate information about the risks borne by the clients. ${ }^{24}$ How would the presence of explicit contracts affect the optimal job design? This section discusses this issue.

In order to accommodate explicit incentives in our model, we can simply "relabel" job 1 as the verifiable job and job 2 as the nonverfiable job. In other words, we assume that $x_{1}$ is a verifiable signal while $x_{2}$ continues to be nonverifiable. Let the piece-rates associated with job 1 under individual accountability be $b_{A}$ and under team accountability be $b_{A 1}$ and $b_{B 1}$. Thus, the firm now chooses the tuple $\left(W_{A}, W_{B}, b_{A}, \beta_{B}\right)$ under individual accountability and the tuple $\left(W_{A}, W_{B}, b_{A 1}, b_{B 1}, \beta_{A 2}, \beta_{B 2}\right)$ under team accountability.

Observe that for given values of $b \mathrm{~s}$ and $\beta \mathrm{s}$, the presence of explicit contracts does not change the agents' incentives in any substantive way (compared to the case where all incentives are implicit). It is merely a matter of relabelling $\beta$ s as $b s$. Thus, it does not affect the $(I R)$ and $(I C)$ constraints. However, the $(D R)$ constraint changes substantially for two reasons. First, instead of both jobs, only the incentives associated with job 2 are now implicitly contracted upon. Second, and more importantly, the presence of explicit contracts changes the firm's punishment payoff. This is due to the fact that the firm can continue to rely on the explicit incentives to elicit some effort from the agents, even on the punishment path (this issue is similar to the one discussed in Baker et al., 1994). Therefore, while discussing below the optimal contracts under individual and team accountability, we will primarily focus on the $(D R)$ constraint.

\footnotetext{
${ }^{22}$ Commercial insurance coverage can often be a complex product, and it may be difficult for the client to assess his exact needs and the best suitable coverage. An important role of the agents is to offer "risk analysis," i.e., to infer the type and degree of risk borne by the client and to advice her on the appropriate coverage.

${ }^{23}$ The success in the match job can be measured by observing how a particular client's account has performed in a given time period. One may expect that if the agents were successful in eliciting the information on the level of risk borne by the client, the insurer would tailor its offered coverage appropriately (and would set the premium rate accordingly) to ensure that the account would be a profitable one. Even though this measure is observable, it is often not verifiable. Insurance claims may take several years to settle. The insurer can manipulate reported performance of the account in a given financial year by using discretion as to when and how to enter the claim record in its books (Wilder, 2002).

24 "Misselling" of products is indeed a major concern in the insurance industry and, in general, in the financial sector (see Indrest and Ottavianni, 2008).
} 
Consider first the case of individual accountability. On the punishment path, agent $B$ reverts back to the static best response and does not exert any effort, i.e., $e_{3}=e_{4}=0$. Consequently, the optimal explicit contract on the punishment path simply solves the following program (after eliminating $W_{A}, W_{B}$ and e using $(I R)$ and $(I C)$ ):

$$
\max _{b_{A}} \underline{\hat{\pi}}^{I} \equiv b_{A} \phi(1+\gamma)-\frac{1}{2} b_{A}^{2}\left(1+\gamma^{2}\right)
$$

The optimal $b_{A}$ thus obtained is $\underline{b}_{A}^{I}=\phi(1+\gamma) /\left(1+\gamma^{2}\right)$, and the optimal punishment payoff is:

$$
\underline{\hat{\pi}}^{I}=\frac{1}{2} \phi^{2} \frac{(1+\gamma)^{2}}{1+\gamma^{2}} .
$$

Now, analogous to the program $\mathcal{P}_{I}$ (i.e., the optimization problem of the firm when both jobs are implicitly contracted, as discussed in the previous section) the firm's optimization problem can be written as (again, after eliminating $W_{A}, W_{B}$ and e using $(I C)$ and $(I R)$ ):

$$
\hat{\mathcal{P}}_{I}:\left\{\begin{array}{cc}
\hat{\pi}_{*}^{I} \equiv \max _{b_{A}, \beta_{B}} \hat{\pi}^{I}\left(b_{A}, \beta_{B}\right)=\phi(1+\gamma)\left(b_{A}+\beta_{B}\right)-\frac{1}{2}\left(1+\gamma^{2}\right)\left(b_{A}^{2}+\beta_{B}^{2}\right) & \\
\text { s.t. } & \frac{\delta}{1-\delta}\left[\hat{\pi}^{I}\left(b_{A}, \beta_{B}\right)-\underline{\hat{\pi}}^{I}\right] \geq \beta_{B} .
\end{array}\right.
$$

It is important to note the following about $\hat{\mathcal{P}}_{I}$ : As before, $\hat{\pi}^{I}\left(b_{A}, \beta_{B}\right)$ is additively separable in $b_{A}$ and $\beta_{B}$. Thus, fixing $\beta_{B}$, the optimal $b_{A}$, say $b_{A}^{*}$, is independent of $\beta_{B}$ and is exactly equal to $\underline{b}_{A}^{I}$. In other words, the firm continues to offer the same explicit contract on both the equilibrium and the punishment paths. An implication of this observation is that $\hat{\mathcal{P}}_{I}$ can be rewritten as:

$$
\begin{gathered}
\qquad \begin{aligned}
\hat{\pi}_{*}^{I} \equiv \max _{\beta_{B}} \hat{\pi}^{I}\left(b_{A}^{*}, \beta_{B}\right)=\phi(1+\gamma)\left(b_{A}^{*}+\beta_{B}\right)-\frac{1}{2}\left(1+\gamma^{2}\right)\left(b_{A}^{* 2}+\beta_{B}^{2}\right) \\
=\underline{\hat{\pi}}^{I}+\phi(1+\gamma) \beta_{B}-\frac{1}{2}\left(1+\gamma^{2}\right) \beta_{B}^{2} \\
\text { s.t. } \quad\left(\widehat{D R}^{I}\right)
\end{aligned}
\end{gathered}
$$

This program is identical to the program $\mathcal{P}_{I}$, except for the fact that the objective function in $\hat{\mathcal{P}}_{I}, \hat{\pi}^{I}\left(b_{A}^{*}, \beta_{B}\right)$ is a linear transformation of the objective function in $\mathcal{P}_{I}$. Thus, one readily obtains the following relationship between the profits associated with the optimal contracts in the two scenarios:

$$
\hat{\pi}_{*}^{I}=\frac{1}{2} \pi_{*}^{I}+\underline{\hat{\pi}}^{I}
$$

The above equation is quite intuitive given the additive separability of the firm's optimization problem with respect to the incentives offered to each of the two agents. There are two key differences between the case where only implicit contracts are offered to the two agents and the case where explicit and implicit incentives are combined: (i) In the latter case, only 
one of the two agents faces an implicit contract and the strength of this implicit contract is the same across the two cases. Hence, he generates a profit of $\pi_{*}^{I} / 2$. (ii) The other agent, the one who is responsible for job 1 , faces an explicit contract that is the same on the punishment path and on the equilibrium path. Hence, he always generates a profit of $\underline{\hat{\pi}}^{I}$.

The same logic holds in the case of team accountability. But of course, the punishment payoff under team accountability is different from its individual accountability counterpart. On the punishment path under team accountability, both agents exert effort only in response to the explicit incentives. Thus, $e_{3}=e_{4}=0$ as job 2 is compensated only through implicit contracts. For efforts associated with job 1, the $(I C)$ constraints for the agents imply that $e_{1}=b_{A 1}$, and $e_{2}=\gamma b_{B 1}$. Therefore, analogous to the case of individual accountability, the optimal explicit contract on the punishment path simply solves the following program (after eliminating $W_{A}, W_{B}$, and e using $(I R)$ and $\left.(I C)\right)$ :

$$
\max _{b_{A 1}, b_{B 1}} \underline{\hat{\pi}}^{T} \equiv \phi\left(b_{A 1}+\gamma b_{B 1}\right)-\frac{1}{2}\left(b_{A 1}^{2}+\gamma^{2} b_{B 1}^{2}\right)
$$

The optimal $b_{A}$ and $b_{B}$ thus obtained are $\underline{b}_{A 1}^{T}=\phi$, and $\underline{b}_{B 1}^{T}=\phi / \gamma$, and the optimal punishment payoff is:

$$
\underline{\hat{\pi}}^{T}=\phi^{2}
$$

Now, analogous to the case of individual accountability (i.e., as given in equation (10)), the equilibrium payoff under team accountability, say, $\hat{\pi}_{*}^{T}$, is given as follows:

$$
\hat{\pi}_{*}^{T}=\frac{1}{2} \pi_{*}^{T}+\underline{\hat{\pi}}^{T}
$$

Equations (10) and (12) offer a simple characterization of the firm's equilibrium payoff under team and individual accountability when explicit contracts are combined with implicit incentives. Using these relationships, the following proposition shows that the optimal job design no longer follows a cut-off rule in the presence of explicit incentives.

Proposition 3. If $\gamma$ is sufficiently large, team accountability is optimal for all values of $r$. Else, there exist two values of $r$, say $r_{1}$ and $r_{2}$, such that individual accountability is optimal for all $r \in\left[r_{1}, r_{2}\right]$, and team accountability is strictly optimal otherwise.

The intuition behind this result is similar to the cut-off result discussed in Proposition 1, particularly when $r$ is not too large. For $r$ sufficiently small (i.e., $\delta$ sufficiently large), the firm's reputational capital is sufficiently large. Thus, the firm can offer strong implicit incentives even under team accountability. Consequently, team accountability becomes optimal because it overcomes the multitasking problem. In contrast, for moderate values of $r$, individual accountability dominates. This is due to the fact that for a moderate $r$, the firm has some reputational capital that allows it to offer implicit incentives. In such a scenario, the implicit incentives are sharper under individual accountability. This is because the firm only needs to promise a bonus payment to one of the two agents, and hence, can credibly promise a larger bonus amount than it could if it were to promise a bonus payment to each of the two agents (as is the case with team accountability). When the multitasking problem is not too large (i.e., moderate $\gamma$ ), this incentive effect outweighs the multitasking problem associated with 
individual accountability. But what drives the optimality of teams for sufficiently large $r$ ? For $r$ sufficiently large (i.e., $\delta$ sufficiently small), the firm has little reputation concerns, and hence, the implicit incentives are infeasible under both types of job designs. The firm's profit under team accountability is higher because the explicit incentive can elicit more search effort under team setting by mitigating the multitasking problem.

However, if the multitasking problem is sufficiently large, then even for a moderate $r$, the stronger implicit incentives under individual accountability need not be enough to compensate for the associated multitasking problem. In this case, team accountability remains optimal for all values of $r$.

Figure 2 depicts an illustrative example of the case where individual accountability is optimal for intermediate values of $r$.

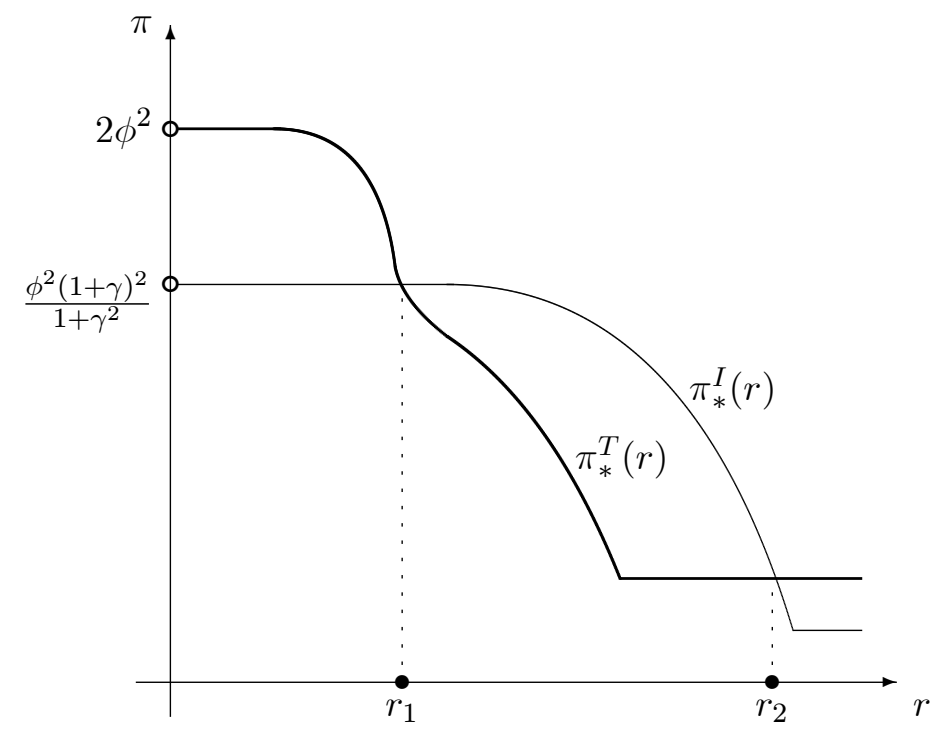

Figure 2. The maximal profits under team and individual accountability in the presence of explicit incentives: The case where $\gamma$ is small (team is optimal for $r<r_{1}$ and $r>r_{2}$ )

The following proposition presents a comparative statics with respect to the $\gamma$.

Proposition 4. Both $r_{1}$ and $r_{2}$ are increasing in $\gamma$. Moreover, there exist two threshold values of $\gamma$, say $\gamma$ and $\bar{\gamma}$, such that (i) for $\gamma<\gamma, r_{2}-r_{1}$ (the size of the interval for which individual accountability is optimal) may increase or decrease in $\gamma$, (ii) for $\underline{\gamma}<\gamma<\bar{\gamma}, r_{2}-r_{1}$ decreases in $\gamma$, and (iii) for all $\gamma>\bar{\gamma}$, team accountability is always optimál.

The proposition above suggests that if $\gamma$ is not too small to begin with, team accountability becomes more likely to be the optimal job design as the extent of multitasking problem $(\gamma)$ increases. Indeed, when $\gamma$ is sufficiently large, individual accountability is never optimal. This result is similar in spirit to Proposition 2, but with one caveat: for sufficiently low $\gamma$, an increase in the extent of the multitasking problem may favor individual accountability. This happens for the following reason. An increase in $\gamma$ has two effects on the firm's payoff: (i) Incentive effect: It increases the effort level in the $\gamma$-tasks as the marginal benefit of task 
2 and task 4 increases with $\gamma$. This effect favors both individual and team accountability. ${ }^{25}$ However, it is a priori unclear under which job design this effect is more pronounced. (ii) Multitasking effect: It accentuates the multitasking problem, and, therefore, increases the loss of efficiency due to the misallocation of effort across tasks. This effect works in favor of team accountability. When $\gamma$ is sufficiently small, the loss of surplus due to the multitasking problem is small. Thus, if the underlying parameters are such that the incentive effect is significantly stronger under individual accountability, it may dwarf the multitasking effect. Therefore, when $\gamma$ is sufficiently small to begin with, an increase in $\gamma$ may favor individual accountability.

We conclude this section with the following observation: our assumption that the job design is irreversible even on the punishment path may appear even more stringent in the current setting since on the punishment path, we allow the firm to change its explicit contract. So, one may ask: if the firm can rearrange the optimal explicit contract, why cannot it change its job design on the punishment path? The key motivation behind maintaining this assumption is that changing job design might be costly and time consuming (e.g., workers may need to be trained for their new tasks) and therefore, the firm may not be able to change its job design as promptly as it can change its contracts with the workers. Having said that, it is worth emphasizing that the statement of Proposition 3 remains unchanged (but of course, the cutoff values $r_{1}$ and $r_{2}$ will be different) if we relax the "irreversibility of job design" assumption. Overall, team accountability becomes more likely to be the optimal job design if this assumption is relaxed. The proof of Proposition 3 when job design is indeed reversible on the punishment path is given in the appendix.

\section{ExTENSIONS AND Discussion}

The results discussed in the previous sections (Propositions 1 and 3) offer a sharp characterization of the optimal job design. However, as noted in description of the basic model, these results are derived under a technology specification that rules out any interaction between efforts in different tasks. To what extent are the key results robust to an alternative technology specification that allows interaction between efforts? Can one generalize our result in a model with an arbitrary number of jobs, tasks, and agents? Also, does the main result continues to hold if a more general class of contracts becomes feasible? This section discusses the robustness of our main results to each of these two issues. It also explores some of the salient empirical implications of our results.

5.1. Substitutability between efforts in different tasks. Many of the multitasking models in the current literature assume that efforts in different tasks are substitutes in the agents' cost functions (e.g., Holmström and Milgrom, 1991). In these models, substitutability of effort is one of the main sources of the multitasking problem. In contrast, in the model used above, the cost of effort is assumed to be additively separable. While such additive separability improves the analytical tractability of the model, it rules out any room for substitutability between efforts in different tasks. However, it turns out that the key insights of our basic model (as discussed in the context of Proposition 1) continue to hold, even if one allows for substitutability between efforts in the agents' cost function. The purpose of this subsection is to illustrate this point.

In order to introduce substitutability between efforts, we insert an interaction term in the agents' cost functions. Keeping all other aspects of the model unchanged, we assume that the total cost of effort for agent $k \in\{A, B\}$ when he is assigned to tasks $i$ and $j$ is

\footnotetext{
${ }^{25}$ It is straightforward to see this effect from the agents' $(I C)$ constraints. For example, for agent $A$, under individual accountability, $\left(I C_{A}^{I}\right)$ implies $e_{2}=\gamma \beta_{A}$ and $\left(I C_{A}^{T}\right)$ implies $e_{4}=\gamma \beta_{A 2}$. Thus, in equilibrium, an increase in $\gamma$ increases both $e_{2}$ and $e_{4}$.
} 


$$
c\left(e_{i}, e_{j}\right)=\frac{1}{2} e_{i}^{2}+\frac{1}{2} e_{j}^{2}+\theta e_{i} e_{j}
$$

where $\theta \in(0,1]$ is a measure of substitutability between efforts in different tasks. Thus, agent $A$ 's total cost of effort under individual accountability (when he is assigned to tasks 1 and 2 ) is $c\left(e_{1}, e_{2}\right)=e_{1}^{2} / 2+e_{2}^{2} / 2+\theta e_{1} e_{2}$, and under team accountability (when he is assigned to tasks 1 and 4$)$ is $c\left(e_{1}, e_{4}\right)=e_{1}^{2} / 2+e_{4}^{2} / 2+\theta e_{1} e_{4}$ (similarly for agent $B$ ). Note that when $\theta=0$, we revert back to the basic model. And at the other extreme, when $\theta=1$, the efforts in the two tasks become perfect substitutes. The following proposition highlights that when the extent of substitutability is not too large, the key insights of the basic model continue to hold.

Proposition 5. For $\theta>1 / \gamma$, team accountability is always optimal. Else, there exists a value for $r$, say $\hat{r}$, such that team accountability is optimal for all $r<\hat{r}$, and individual accountability is optimal otherwise.

The proposition above is similar in spirit to Proposition 1 with one important addition: for $\theta$ sufficiently large, team accountability is always optimal. The intuition behind this finding is as follows: Recall that the parameter $\theta$ measures the extent of substitutability between efforts in different tasks. Thus, when $\theta$ is high, high effort in one task significantly increases the marginal cost of effort in the other task. This effect makes the multitasking problem more severe. The agent now has a stronger incentive to focus on the " $\gamma$-task" (at the expense of the other task) not only because the effort in the $\gamma$-task has a higher marginal impact on the performance signal, but also because a high effort in the $\gamma$-task makes the effort in the other task more costly.

In such a scenario, team accountability is even more effective (compared to the case in the basic model) in eliciting higher efforts in both tasks. As discussed before, team accountability helps the firm overcome the multitasking problem that emanates from the fact that the efforts in the two tasks have different marginal impacts on the performance signal (modelled through the parameter $\gamma$ ). In addition, by varying the incentives for effort in the two tasks, team accountability can also offset the effort substitution towards the " $\gamma$-task" that emanates from the substitutability of effort in the agents' cost function (modelled through the parameter $\theta$ ). For example, the firm can leave the incentives for the " $\gamma$-task" unchanged, but increase the incentive for the other task ("non- $\gamma$-task") to compensate the agent for the higher marginal cost of effort in the "non- $\gamma$-task" that stems from the high effort in the " $\gamma$-task." This effect makes team accountability more desirable when efforts are strong substitutes in the agents' cost function.

Corollary 1. When efforts are perfect substitutes in the agents' cost function (i.e., when $\theta=1)$, team accountability is always optimal.

This observation follows directly from Proposition 5 (hence we omit the proof in the Appendix). As $\gamma>1$, when $\theta=1$, the condition $\theta>1 / \gamma$ is trivially satisfied, and this ensures the optimality of team accountability irrespective of the firm's reputation concerns 
5.2. Generalization to multiple jobs, tasks, and agents. The basic intuition behind our finding continues to hold in a more general setup with an arbitrary number of jobs, tasks, and agents. Suppose there are $N$ agents, $N$ jobs, and each job involves $N$ tasks. Denote $e_{j k}$ as the effort exerted on task $k$ in job $j$. Let $V(\mathbf{e})=\phi \sum_{k, j} e_{j k}$ and $\operatorname{Pr}\left(x_{j}=1 \mid \mathbf{e}\right)=\sum_{k} \gamma_{k} e_{j k}$, and $\gamma_{k} \neq \gamma_{k^{\prime}}$ for some tasks $k$ and $k^{\prime}$ in job $j$. We continue to maintain the assumption that the total cost of effort to an agent is $\sum e_{j k}^{2} / 2$ (where the sum is taken over all the tasks that have been assigned to the agent). Observe that the key trade-off between multitasking and the sustenance of implicit contracts continues to prevail. Effort in each task has the same marginal impact on the firm's value, but an agent assigned to job $j$ will continue to exert more effort on the tasks that have higher impact on the performance measure $x_{j}$ (i.e., that have higher $\gamma$ coefficient in the $\operatorname{Pr}\left(x_{j}=1 \mid \mathbf{e}\right)$ function). As before, under team accountability, the firm can assign each of the $k$ tasks to $k$ different agents and fine-tune its incentives to overcome the multitasking problem. But, the firm now has to commit to a larger bonus pool, because it has to make separate payments to each of the $k$ different agents to elicit effort in all of the $k$ tasks.

In fact, the cut-off result discussed in Proposition 1 continues to hold in a general setting. For analytical tractability, suppose that there are $K_{1}$ jobs that are "non- $\gamma$ jobs" (i.e., $\gamma_{K}=1$ for $K=1,2, \ldots, K_{1}$ ) and $K_{2}$ " $\gamma$-jobs" (i.e., $\gamma_{K}=\gamma$ for $K=K_{1}+1, \ldots, K$ ), where $K_{1}+K_{2}=N$. Proposition 6 shows that team accountability is still optimal only for the firms with sufficiently high reputation concerns (low $r$ ).

Proposition 6. There exists a value of $r$, say $r^{* *}$, such that team accountability is strictly optimal if and only if $r<r^{* *}$.

Two issues are worth mentioning in this regard. First, one may ask the following: if there are arbitrary number of jobs, tasks, and agents (i.e., the number of jobs, tasks and agents need not be equal to each other), under what condition does team accountability necessarily solve the multitasking problem (i.e., elicit the first-best effort in all tasks in all jobs)? The answer to this question directly follows from Corts (2007). He shows that team accountability can elicit first-best effort allocation as long as there are enough agents so that in order to elicit effort in all tasks in all jobs, the firm does not have to assign any agent to more tasks than there are jobs. ${ }^{26}$ The intuition is that, in order to overcome the multitasking problem, the team must generate a sufficiently rich set of performance measures for all agents. If an agent is assigned to more tasks than there are jobs, then it must be the case that his performance in at least two tasks is rewarded based on the outcome of a single job. Consequently, the multitasking problem prevails.

The second issue concerns the comparative statics predictions. In the basic model, if one increases the multitasking problem by increasing $\gamma$, team accountability becomes more likely to be the optimal. In the general model one can also increase the extent of the multitasking problem by increasing the number of tasks in each job (i.e., increase $K$ ). Does an increase in $K$ also favor team accountability? It may not. The reason is that an increase in the number of tasks cuts both ways: it aggravates the multitasking problem under individual accountability and favors team accountability, but it also requires more agents to be assigned in the same job (albeit in different tasks) to overcome the aggravated multitasking problem. This calls for an increased bonus pool (equivalently, a more severe free-riding problem) in teams that the firm may not be able to commit to.

\footnotetext{
${ }^{26}$ Note that this condition is trivially satisfied in our basic model as well as in this general discussion. In the general case, the number of jobs, tasks and agents are all equal to $N$. The basic model is a special case of the general model where $N=2$.
} 
5.3. General class of contracts. In the model we have considered a class of contracts that is additively separable across jobs. While nonseparable compensations schemes may have their own disadvantages (such as incentives for sabotage or collusion among coworkers) additively separable contracts rule out certain interesting compensation schemes such as relative performance evaluations. For example, under individual accountability, agent $A$ 's bonus following a success in job 1 may depend on whether agent $B$ is also successful (Levin (2002) shows that such contracts can be optimal in some settings).

The qualitative nature of our results does not change even if we allow for such relative performance evaluations. To see this, consider a general contract under individual accountability that is defined as follows: the contract offered to agent $i \in\{A, B\}$ consists of a fixed wage $W_{i}$ and bonus payments $\beta_{i \mathbf{x}}$ given the performance outcome in jobs 1 and $2, \mathbf{x}=\left(x_{1}, x_{2}\right){ }^{27}$ Now, the agents' optimization problems are given by

$$
\max _{e_{1}, e_{2}} W_{A}+\sum_{\mathbf{x}} \beta_{A \mathbf{x}} \operatorname{Pr}(\mathbf{x} \mid \mathbf{e})-c\left(e_{1}\right)-c\left(e_{2}\right)
$$

and

$$
\max _{e_{3}, e_{4}} W_{B}+\sum_{\mathbf{x}} \beta_{B \mathbf{x}} \operatorname{Pr}(\mathbf{x} \mid \mathbf{e})-c\left(e_{3}\right)-c\left(e_{4}\right)
$$

where $\operatorname{Pr}(\mathbf{x} \mid \mathbf{e})$ is the probability of outcome $\mathbf{x} \in\{(0,0),(0,1),(1,0),(1,1)\}$ given $\mathbf{e}=\left(e_{1}, e_{2}, e_{3}, e_{4}\right)$. Solving these problems one finds that the optimal effort choice of agent $A$ is: ${ }^{28}$

$$
e_{1}=\frac{\beta_{A 10}+\left(1+\gamma^{2}\right) \beta_{B 01}\left(\beta_{A 11}-\beta_{A 10}\right)}{1-\left(1+\gamma^{2}\right)^{2}\left(\beta_{A 11}-\beta_{A 10}\right)\left(\beta_{B 11}-\beta_{B 01}\right)} \text { and } e_{2}=\gamma e_{1},
$$

and that of agent $B$ is:

$$
e_{3}=\frac{\beta_{B 01}+\left(1+\gamma^{2}\right) \beta_{A 10}\left(\beta_{B 11}-\beta_{B 01}\right)}{1-\left(1+\gamma^{2}\right)^{2}\left(\beta_{A 11}-\beta_{A 10}\right)\left(\beta_{B 11}-\beta_{B 01}\right)} \text { and } e_{4}=\gamma e_{3} .
$$

Two issues are important to note in the solutions above. First, the multitasking problem continues to hold under individual accountability. For any arbitrary contract, $e_{1}$ and $e_{2}$ cannot be perfectly aligned (similarly for $e_{3}$ and $e_{4}$ ). Second, relative performance evaluation might be optimal when the firm has little reputation concerns. For example, from (13) one finds $\partial e_{1} / \partial \beta_{A 10}=1-\left(1+\gamma^{2}\right) \beta_{B 01}$ when $\beta_{A 10}=\beta_{A 11}$ (i.e., when the initial contract does not consider relative performances). But $\beta_{B 01}$ must be less than the total bonus, say $\beta$, that the firm can credibly commit to. Hence, when $\beta$ is sufficiently small and $\beta_{A 10}=\beta_{A 11}$, $\partial e_{1} / \partial \beta_{A 10}>0$. In other words, when the firm does not have the credibility to promise a large bonus, it is more efficient to pay a higher bonus to a successful agent $A$ when agent $B$ fails than when agent $B$ succeeds. An analogous argument holds for agent $B$.

Note that this finding does not affect the qualitative nature of our main result. If we allow for relative performance contract, the firm's payoff under individual accountability is (weakly) larger when $\delta$ is low (i.e., when the size of the total bonus pool is low). But for low $\delta$, individual accountability dominates team accountability, even under the additively

\footnotetext{
${ }^{27}$ Thus, because there are four possible outcomes of the performance measures in jobs 1 and 2 , there are four possible bonuses for agent $A: \beta_{A 11}$ (if $x=(1,1)$ ), $\beta_{A 10}$ (if $x=(1,0)$ ), $\beta_{A 01}$ (if $x=(0,1)$ ) and $\beta_{A 00}$ (if $x=(0,0))$. The same holds for agent $B$.

${ }^{28}$ The associated first-order conditions reveal that the optimal effort of an agent, say agent $A$, is driven only by the differences in the bonus payments $\beta_{A 10}-\beta_{A 00}$ and $\beta_{A 11}-\beta_{A 01}$ (similarly for agent $B$ ). Thus, in the optimal contract, we will normalize $\beta_{A 01}=\beta_{A 00}=\beta_{B 10}=\beta_{B 00}=0$.
} 
separable compensation scheme. Therefore, the feasibility of relative performance evaluation would only reinforce our findings.

One can also consider a more general contract under team accountability where the firm promises an additional bonus when both jobs are successful. For example, the firm may promise a bonus, say $\beta_{A}$, to agent $A$ in addition to the bonuses $\beta_{A 1}$ (paid when job 1 is successful) and $\beta_{A 2}$ (paid when job 2 is successful). Similarly, the firm can promise an additional bonus $\beta_{B}$ to agent $B$.

However, the qualitative nature of our main finding - that team accountability is optimal for high $\delta$ and individual accountability is optimal for low $\delta$-continues to hold even if such contracts are in place. When the firm has low reputation concerns, it is optimal to set $\beta_{A}$ and $\beta_{B}$ to zero because such incentives are less efficient in eliciting effort than the more direct bonus incentives $\beta_{A 1}, \beta_{A 2}, \beta_{B 1}$ and $\beta_{B 2}$. Because $\beta_{A}$ and $\beta_{B}$ are offered only if both jobs are successful, the agent will collect them only with probability $\operatorname{Pr}\left(x_{1} \mid \mathbf{e}\right) \times \operatorname{Pr}\left(x_{2} \mid \mathbf{e}\right)$. When the firm cannot promise a strong implicit contract, efforts will be small and the marginal impact of effort on the product $\operatorname{Pr}\left(x_{1} \mid \mathbf{e}\right) \times \operatorname{Pr}\left(x_{2} \mid \mathbf{e}\right)$ would be significantly smaller than the marginal impact of effort on each of these individual probabilities. Hence, the firm gets more effort per dollar of bonus when the bonus is offered as a direct incentives (i.e., $\beta_{A 1}, \beta_{A 2}, \beta_{B 1}$ and $\beta_{B 2}$ ). Consequently, when the firm's reputational capital is very low (or when $\delta$ is small), not allowing for such bonus is without loss of generality and individual accountability continues to dominate team accountability when $\delta$ is small.

5.4. Empirical implications. Propositions 1 and 3, along with their corollaries, have important empirical implications. They highlight the fact that when bonus incentives are used in a multitasking environment, a key parameter that affects the firm's job design decision (and hence, its performance) is the firm's discount factor, $\delta$. Furthermore, these results suggest that the relationship between $\delta$ and the optimal job design crucially depends on the type(s) of incentives that are in place (i.e., explicit and/or implicit). When only implicit incentives are feasible, team accountability is more likely for firms with high $\delta$. In contrast, when both implicit and explicit contracts are in place, team accountability becomes more likely for firms with either sufficiently low or sufficiently high $\delta$. The extent of the multitasking problem also plays an important role. However, the comparative statics with respect to $\gamma$ is relatively straightforward: for a given $\delta$, team accountability is more likely when the multitasking problem is more severe (i.e., when $\gamma$ is large).

A potential challenge in testing these predictions is that the appropriate empirical measures of $\gamma$ and $\delta$ in a given industry might be difficult to obtain. Also, in many cases a firm's job design decision may be an artifact of the underlying production technology rather than a strategic choice made by the firm. However, the results of our model can be put to the test in the context of certain industries where these measures are perhaps easier to obtain. The mutual funds industry may be one such candidate. One can use a fund's liquidation probability as a measure of $\delta$ (Getmansky et al., 2004). But finding an empirical measure of $\gamma$ is more challenging. Information on the types of assets a fund is invested in may be indicative of the extent of the multitasking the fund's managers are exposed to. For example, a fund that is primarily invested in government bonds and treasury bills faces lower risks compared to a fund that is entirely invested in the stock market. One may argue that the manager of the latter fund faces a higher multitasking problem because she not only is responsible for increasing the returns of the investment but also has to pay close attention to the underlying risks that the fund is bearing. ${ }^{29}$ As discussed before, mutual funds often classify themselves as "comanaged" or "team managed" funds, where a group of employees are jointly responsible

\footnotetext{
${ }^{29}$ However, one must be careful of a potential endogeneity problem stemming from the fact that the type of assets in which the fund is invested is also a choice variable for the firm.
} 
for the performance of a set of funds. If one can interpret sole managed funds as individual accountability and team managed funds as team accountability, data on mutual funds may be used to test the predictions discussed above. For example, our result suggests that, if implicit contracts are the only incentive device used by the funds, one may expect to find that funds with low liquidation probabilities (high $\delta$ ) and relatively risky portfolio (high $\gamma$ ) are team-managed, whereas funds with high liquidation probabilities (low $\delta$ ) and relatively safe portfolio (low $\gamma$ ) are individually managed.

Several authors have studied the relative profitability of the individual- and team-managed funds (Prather and Middleton, 2002; Massa, 2008) ${ }^{30}$ but empirical studies on the impact of the firms' reputation concerns on job design are scarce. Our findings indicate that in the presence of multitasking and implicit contracts, a firm's discount factor can indeed play a key role in its job design decision. Therefore, in such an environment, it might be important that an empirical investigation of the profitability of different job designs controls for the firms' reputation concerns in order to avoid any potential selection bias.

\section{Conclusion}

In many industries, firms often adopt team accountability, even when individual accountability remains a technologically viable option. This article highlights how team accountability may emerge endogenously in a multitasking environment, where the firm relies on implicit contracts, i.e., bonus payments. In the presence of implicit contracts, team accountability involves an interesting trade-off: it alleviates the multitasking problem but weakens the implicit incentives. The contribution of this article is to formalize this trade-off and to draw out its implications on the firms' optimal job design policy.

The key result is that the optimal job design follows a cut-off rule. Only the firms with high enough reputation concerns (i.e., discount factor) opt for team accountability. The more acute the multitasking problem is, the more likely it is that the firm would opt for team accountability. However, when, in addition to implicit contracts, explicit pay-perperformance contracts are also feasible, the cut-off rule stated above is no longer optimal. In such a scenario, firms with both sufficiently high and sufficiently low reputation concerns opt for team accountability, and firms with moderate reputation concerns opt for individual accountability.

Overcoming the multitasking problem need not be the only driver of a firm's job design decision. For example, team accountability may emerge to manipulate career concerns of the agents by obscuring their individual contributions to the project's overall performance (Massa et al., 2008). Teams may also originate from the need to facilitate cooperation within organization (Shaw and Schneier, 1995). But the main contribution of this article is in extending our understanding of how job design interacts with implicit contracts and how firms may profit from adopting team accountability in a multitasking environment when they must rely on implicit incentives.

\section{APPENDIX}

Proof of Lemma 2. The optimal profit under individual accountability is obtained by solving program $\mathcal{P}_{I}$. Let $\widehat{\pi}^{I}(\beta)=2\left[\phi(1+\gamma) \beta-\frac{1}{2}\left(1+\gamma^{2}\right) \beta^{2}\right]$, which is the function being maximized in $\mathcal{P}_{I}$; and note that that constraint $D R^{I}$ in $\mathcal{P}_{I}$ can be written as $R^{I}(\beta):=$ $\phi(1+\gamma) \beta-\frac{1}{2}\left(1+\gamma^{2}\right) \beta^{2} \geq r \beta$. The remainder of the proof is given by the following steps.

\footnotetext{
${ }^{30}$ However, as noted earlier, in both of these articles factors other than the mutlitasking problem may also drive the choice between individual and team accountability.
} 
Step 1. Suppose that $r \leq \phi(1+\gamma) / 2$. In this case, the $D R^{I}$ constraint is satisfied when $\beta=\phi(1+\gamma) /\left(1+\gamma^{2}\right)$, which is the (unconstrained) $\arg \max \widehat{\pi}^{I}(\beta)$. Thus, in this case, $\beta=\phi(1+\gamma) /\left(1+\gamma^{2}\right)$ must be the solution to $\mathcal{P}_{I}$.

Step 2. Suppose that $r \geq \phi(1+\gamma)$. In this case, $R^{I \prime}(0) \leq r$. Because $R^{I}(0)=0$ and $R^{I}$ is concave, this implies that $R^{I}(\beta)<r \beta$ for all $\beta>0$, and the only value of $\beta$ that satisfies the $D R^{I}$ constraint is $\beta=0$. Thus, in this case, $\beta=0$ is the solution to $\mathcal{P}_{I}$.

Step 3. Suppose that $\phi(1+\gamma) / 2<r<\phi(1+\gamma)$. Because $r>\phi(1+\gamma) / 2$, the $D R^{I}$ constraint is violated for all $\beta \geq \phi(1+\gamma) /\left(1+\gamma^{2}\right)$. Thus, from direct inspection of the $D R^{I}$ constraint and $\widehat{\pi}^{I}(\beta)$, it immediately follows that the solution to $\mathcal{P}_{I}$ is the highest value of $\beta$ that satisfies the $D R^{I}$ constraint. Because $r<\phi(1+\gamma), R^{I^{\prime}}(0)>r$. This, together with the fact that $R^{I}$ is concave and $R^{I}(\beta)<r \beta$ for all $\beta \geq \phi(1+\gamma) /\left(1+\gamma^{2}\right)$, implies that the highest value of $\beta$ that satisfies the $D R^{I}$ constraint is given by the condition $R^{I}(\beta)=r \beta$. Thus, in this case, $\beta=(2 \phi(1+\gamma)-2 r) /\left(1+\gamma^{2}\right)$ is the solution to $\mathcal{P}_{I}$.

To obtain $\pi_{*}^{I}(r)$ as given in the statement of the Lemma, simply plug the solutions obtained for $\beta$ in Steps $1-3$ in $\widehat{\pi}^{I}(\beta)$.

Proof of Lemma 3. The optimal profit under team accountability is obtained by solving problem $\mathcal{P}_{T}$. We solve this problem in two steps. First, for a given value of total bonus payments $\beta=\beta_{A 1}+\beta_{B 1}$, we characterize the optimal individual bonus payments $\beta_{A 1}$ and $\beta_{B 1}$. Second, given the optimal $\beta_{A 1}$ and $\beta_{B 1}$ as a function of $\beta$, we find the optimal $\beta$ that the firm can sustain.

The optimal individual bonus payments $\beta_{A 1}$ and $\beta_{B 1}$ given total bonus $\beta$, solve the following problem:

$$
\begin{aligned}
R^{T}(\beta):= & \max _{\beta_{A 1}, \beta_{B 1}} \phi\left(\beta_{A 1}+\gamma \beta_{B 1}\right)-\frac{1}{2}\left(\beta_{A 1}^{2}+\gamma^{2} \beta_{B 1}^{2}\right) \\
\text { s.t. } & \beta_{A 1}+\beta_{B 1} \leq \beta, \beta_{A 1} \geq 0, \text { and } \beta_{B 1} \geq 0
\end{aligned}
$$

Note the $R^{T}(\beta)$ denotes the reputational capital that is achievable given total bonus $\beta$. Using the Kuhn-Tucker optimization method, we obtain that the solution to this problem is as given in $(7)$ :

$$
\begin{array}{lll}
\beta_{A 1}^{T^{*}}=0, & \beta_{B 1}^{T^{*}}=\beta & \text { if } \beta<\phi \frac{\gamma-1}{\gamma^{2}} \\
\beta_{A 1}^{T^{*}}=\frac{\gamma^{2} \beta-\phi(\gamma-1)}{1+\gamma^{2}}, & \beta_{B 1}^{T^{*}}=\frac{\beta+\phi(\gamma-1)}{1+\gamma^{2}} & \text { if } \phi \frac{\gamma-1}{\gamma^{2}} \leq \beta<\phi \frac{\gamma+1}{\gamma} \\
\beta_{A 1}^{T^{*}}=\phi, & \beta_{B 1}^{T^{*}}=\frac{\phi}{\gamma} & \text { if } \beta=\phi \frac{\gamma+1}{\gamma} .
\end{array}
$$

This solution implies that

$$
R^{T}(\beta)= \begin{cases}\phi \gamma \beta-\frac{1}{2} \gamma^{2} \beta^{2} & \text { if } \beta<\phi \frac{\gamma-1}{\gamma^{2}} \\ \left(2 \phi \gamma \beta(1+\gamma)+\phi^{2}(1-\gamma)^{2}-\gamma^{2} \beta^{2}\right) / 2\left(1+\gamma^{2}\right) & \text { if } \phi \frac{\gamma-1}{\gamma^{2}} \leq \beta<\phi \frac{\gamma+1}{\gamma} \\ \phi^{2} & \text { if } \beta \geq \phi \frac{\gamma+1}{\gamma}\end{cases}
$$

Now the firm's optimization problem boils down to: $\pi_{*}^{T} \equiv \max 2 R^{T}(\beta)$ s.t. $R^{T}(\beta) \geq r \beta$. Using the same procedure that is used to solve program $\mathcal{P}_{I}$ (see the proof of Lemma 2), we obtain that the solution to this problem is

$$
\beta_{*}^{T}(r)= \begin{cases}\frac{1}{\gamma} \phi(\gamma+1) & \text { if } r \leq \frac{\phi \gamma}{\gamma+1} \\ \frac{1}{\gamma^{2}} K(r) & \text { if } \frac{\phi \gamma}{\gamma+1}<r \leq \frac{1}{2} \phi(\gamma+1) \\ \frac{2}{\gamma^{2}}(\phi \gamma-r) & \text { if } \frac{1}{2} \phi(\gamma+1)<r<\phi \gamma \\ 0 & \text { if } r \geq \phi \gamma\end{cases}
$$


where the function $K(r)=\phi \gamma(1+\gamma)-r\left(1+\gamma^{2}\right)+\left[\left(\phi \gamma(1+\gamma)-r\left(1+\gamma^{2}\right)\right)^{2}+\gamma^{2}(1-\gamma)^{2} \phi^{2}\right]^{1 / 2}$. To obtain $\pi_{*}^{T}(r)$ as given in the statement of the Lemma, plug this solution in $2 R^{T}(\beta)$.

Proof of Proposition 1. The proof is given by the following steps.

Step 1. We first show that for $r \in[\phi(1+\gamma) / 2, \phi \gamma], \pi_{*}^{I}(r)$ and $\pi_{*}^{T}(r)$ cannot intersect. To prove this fact we simply note the following. First, $\left|\partial \pi_{*}^{I} / \partial r\right|<\left|\partial \pi_{*}^{T} / \partial r\right|$ for $r \in[\phi(1+\gamma) / 2, \phi \gamma]$. Second, $\pi_{*}^{T}(\phi(1+\gamma) / 2)=\phi^{2}\left(1-\gamma^{-2}\right)<\phi^{2}(1+\gamma)^{2} /\left(1+\gamma^{2}\right)=$ $\pi_{*}^{I}(\phi(1+\gamma) / 2)$.

Step 2. For $r \in[\phi \gamma /(1+\gamma), \phi(1+\gamma) / 2], \pi_{*}^{I}(r)$ and $\pi_{*}^{T}(r)$ must intersect at an unique point. To see this, note that for all $r \in[\phi \gamma /(1+\gamma), \phi(1+\gamma) / 2], \pi_{*}^{I}(r)=\phi^{2}(1+\gamma)^{2} /\left(1+\gamma^{2}\right)$. But $\partial \pi_{*}^{T} / \partial r<0$ for $r \in[\phi \gamma /(1+\gamma), \phi(1+\gamma) / 2]$ and $\pi_{*}^{T}(\phi(1+\gamma) / 2)=\phi^{2}\left(1-\gamma^{-2}\right)<$ $\phi^{2}(1+\gamma)^{2} /\left(1+\gamma^{2}\right)<2 \phi^{2}=\pi_{*}^{T}(\phi \gamma /(1+\gamma))$. So, by Mean Value Theorem, there must exist a value of $r \in[\phi \gamma /(1+\gamma), \phi(1+\gamma) / 2]$, say $r^{*}$, such that $\pi_{*}^{T}\left(r^{*}\right)=\pi_{*}^{I}\left(r^{*}\right)=\phi^{2}(1+$ $\gamma)^{2} /\left(1+\gamma^{2}\right)$. Finally, $r^{*}$ is unique as $\pi_{*}^{T}$ is monotone for $r \in[\phi \gamma /(1+\gamma), \phi(1+\gamma) / 2]$.

Proof of Proposition 2. As shown in the proof of Proposition 1, $r^{*}$ solves $2 r^{*} K\left(r^{*}\right) / \gamma^{2}=$ $\phi^{2}(1+\gamma)^{2} /\left(1+\gamma^{2}\right)$. This equation has two solution for $r$. Using the fact that $r^{*}<\phi \gamma /(\gamma+1)$, the only admissible solution is $r^{*}=\gamma \phi(1+\gamma)^{2} / 4\left(1+\gamma^{2}\right)$. Observe that $\partial r^{*} / \partial \gamma=$ $\phi\left(1+\gamma\left(4+2 \gamma+\gamma^{2}\right)\right) / 4\left(1+\gamma^{2}\right)^{2}>0$.

Proof of Proposition 3. The proof is similar in spirit to the proof of Proposition 1, and it is given by the following steps.

Step 1. We first show that $\forall r \in[\phi(1+\gamma) / 2, \phi \gamma], \hat{\pi}_{*}^{I}(r)$ and $\hat{\pi}_{*}^{T}(r)$ can intersect at most once. To prove this fact we proceed as follows: For $r \in[\phi(1+\gamma) / 2, \phi \gamma]$ denote $\Delta(r)=$ $\hat{\pi}_{*}^{T}-\hat{\pi}_{*}^{I}$. Thus, if $\hat{\pi}_{*}^{I}(r)$ and $\hat{\pi}_{*}^{T}(r)$ intersect for any value of $r$, say $\hat{r}, \Delta(\hat{r})=0$. Now, $\Delta^{\prime}(r)=-2(2 r+\phi \gamma(\gamma-1)) /\left(\gamma^{2}+\gamma^{4}\right)<0$. Thus, there cannot exist more than one value of $r$ such that $\Delta(r)=0$.

Step 2. Because $\Delta^{\prime}(r)<0$, and $\phi \gamma>\phi(1+\gamma) / 2$, we need to consider three cases: (i) $\Delta(\phi \gamma)>0(\Rightarrow \Delta(\phi(1+\gamma) / 2)>0)$, (ii) $\Delta(\phi \gamma)<0, \Delta(\phi(1+\gamma) / 2)>0$, and (iii) $\Delta(\phi(1+\gamma) / 2)<0(\Rightarrow \Delta(\phi \gamma)>0)$. The rest of the proof characterizes the nature of intersection of $\hat{\pi}_{*}^{I}(r)$ and $\hat{\pi}_{*}^{T}(r)$ in each of these three cases.

Step 3. If $\Delta(\phi \gamma)>0$ (which is the case if $\gamma$ is sufficiently large) $\Delta(r)>0$ for all $r \in[\phi(1+\gamma) / 2, \phi \gamma]$. In this case $\hat{\pi}_{*}^{T}(r)>\hat{\pi}_{*}^{I}(r) \forall r$. To see this, note that $\forall r \in$ $(0, \phi(1+\gamma) / 2], \hat{\pi}_{*}^{I}(r)=\hat{\pi}_{*}^{I}(\phi(1+\gamma) / 2)<\hat{\pi}_{*}^{T}(r)$ (where the last inequality follows from the fact that $\hat{\pi}_{*}^{T}(r)$ is a (weakly) decreasing function in $r$, and $\Delta(\phi(1+\gamma) / 2)>0 \Leftrightarrow$ $\left.\hat{\pi}_{*}^{T}(\phi(1+\gamma) / 2)>\hat{\pi}_{*}^{I}(\phi(1+\gamma) / 2)\right)$. Similarly, $\forall r \in(\phi \gamma, \infty), \hat{\pi}_{*}^{T}(r)=\hat{\pi}_{*}^{T}(\phi \gamma)>\hat{\pi}_{*}^{I}(r)$ (where the last inequality follows from the fact that $\hat{\pi}_{*}^{I}(r)$ is a (weakly) decreasing function in $r$, and $\left.\Delta(\phi \gamma)>0 \Leftrightarrow \hat{\pi}_{*}^{T}(\phi \gamma)>\hat{\pi}_{*}^{I}(\phi \gamma)\right)$.

Step 4. If $\Delta(\phi \gamma)<0$ but $\Delta(\phi(1+\gamma) / 2)>0$, then $\hat{\pi}_{*}^{I}(r)$ and $\hat{\pi}_{*}^{T}(r)$ intersect at exactly two points. By Mean Value Theorem, there exists an value of $r$, say $r_{1} \in[\phi(1+\gamma) / 2, \phi \gamma]$ such that $\Delta\left(r_{1}\right)$. Also, for the argument discussed in Step 3 above, there cannot exist any value of $r<\phi(1+\gamma) / 2$ such that $\Delta(r)=0$. However, there must exist another value of $r \in(\phi \gamma, \infty)$, say $r_{2}$, such that $\Delta\left(r_{2}\right)=0$. The argument is as follows: $\forall r \in(\phi \gamma, \infty)$, $\hat{\pi}_{*}^{T}(r)=\phi^{2}$. But $\hat{\pi}_{*}^{I}(\phi \gamma)>\phi^{2}=\hat{\pi}_{*}^{T}(\phi \gamma)$ (because we start with the premise that $\left.\Delta(\phi \gamma)<0\right)$ and $\hat{\pi}_{*}^{I}(\phi(1+\gamma))=\phi^{2}(1+\gamma)^{2} / 2\left(1+\gamma^{2}\right)<\phi^{2}=\hat{\pi}_{*}^{T}(\phi(1+\gamma))$. As $\hat{\pi}_{*}^{I}$ is continuous and monotone, by Mean Value Theorem, there must exist a unique value of $r$, say $r_{2}$, such that $\hat{\pi}_{*}^{I}\left(r_{2}\right)=\phi^{2}=\hat{\pi}_{*}^{T}\left(r_{2}\right)$.

Step 5. Finally, consider the case where $\Delta(\phi(1+\gamma) / 2)<0$. Because $\Delta^{\prime}(r)<0 \forall r \in$ $[\phi(1+\gamma) / 2, \phi \gamma]$, there cannot exist any value of $r \in[\phi(1+\gamma) / 2, \phi \gamma]$ such that $\Delta(r)=0$. 
However, there must exist a value of $r \in(0, \phi(1+\gamma) / 2)$, say $r_{1}$, such that $\Delta\left(r_{1}\right)=0$. The argument is as follows: For $r \leq \phi \gamma /(1+\gamma), \hat{\pi}_{*}^{T}(r)=2 \phi^{2}>\phi^{2}(1+\gamma)^{2} /\left(1+\gamma^{2}\right)=\hat{\pi}_{*}^{I}(r)$, and by assumption, $\hat{\pi}_{*}^{T}(\phi(1+\gamma) / 2)<\hat{\pi}_{*}^{I}(\phi(1+\gamma) / 2)=\phi^{2}(1+\gamma)^{2} /\left(1+\gamma^{2}\right)$. As $\hat{\pi}_{*}^{T}(r)$ is continuous and monotonically decreasing, by Mean Value Theorem, there exists a unique value of $r$, say $r_{1}$, such that $\Delta\left(r_{1}\right)=0$. Also, by the argument discussed in Step 4, there must exist a unique value of $r \in(\phi \gamma, \infty)$, say $r_{2}$, such that $\Delta\left(r_{2}\right)=0$. This observation completes the proof.

This proposition also continues to hold when job design is assumed to be reversible on the punishment path. The modified proof is given by the following steps:

Step 1: Note that if assumption 2 is relaxed, only $\pi_{*}^{I}$ is affected since the firm would prefer to revert to team accountability on the punishment path. Using the steps discussed in the proof of lemma 2 in the article, one obtains that the firm's payoff under individual accountability is:

$$
\begin{aligned}
& \pi_{*}^{I}(r)= \\
& \begin{cases}\phi^{2}(1+\gamma)^{2} /\left(1+\gamma^{2}\right) & \text { if } r \leq 2 \frac{\phi \gamma}{\gamma+1} \\
{\left[\phi^{2}\left(1+\gamma^{2}\right)+r \phi(1+\gamma)-r^{2}+r \sqrt{(2 \phi-r)(2 \gamma \phi-r)}\right] /\left(1+\gamma^{2}\right)} & \text { if } 2 \frac{\phi \gamma}{\gamma+1}<r<2 \phi \\
\frac{1}{2} \phi^{2}(1+\gamma)^{2} /\left(1+\gamma^{2}\right) & \text { if } r \geq 2 \phi\end{cases}
\end{aligned}
$$

Also recall that the firm's payoff function under team accountability is:

$$
\pi_{*}^{T}(r)= \begin{cases}2 \phi^{2} & \text { if } r \leq \frac{\phi \gamma}{\gamma+1} \\ 2 r K(r) / \gamma^{2} & \text { if } \frac{\phi \gamma}{\gamma+1}<r \leq \frac{1}{2} \phi(\gamma+1) \\ 4 r(\phi \gamma-r) / \gamma^{2} & \text { if } \frac{1}{2} \phi(\gamma+1)<r<\phi \gamma \\ 0 & \text { if } r \geq \phi \gamma\end{cases}
$$

where $K(r)=\phi \gamma(1+\gamma)-r\left(1+\gamma^{2}\right)+\left[\left(\phi \gamma(1+\gamma)-r\left(1+\gamma^{2}\right)\right)^{2}+\gamma^{2}(1-\gamma)^{2} \phi^{2}\right]^{1 / 2}$.

Step 2: Fix $\phi$ at an arbitrary value in $(0,1 / 2)$ (note that by assumption 1 and the fact that $\gamma>1, \phi \in(0,1 / 2))$. Now, depending on the value of $\gamma$ we need to consider multiple cases (and subcases):

Step 2a (Case 1: $\gamma \geq 3$ ): In this case $\frac{1}{2} \phi(\gamma+1)>2 \phi$. Several subcases must be considered:

Case 1.1: $\gamma$ is high enough so that $\pi_{*}^{T}(r)>\pi_{*}^{I}(r)$ for all $r$. Clearly in this case, the functions never cross and team is always better.

Case 1.2: $\gamma$ is such that $\pi_{*}^{T}\left(2 \phi \frac{\gamma}{\gamma+1}\right)>\pi_{*}^{I}\left(2 \phi \frac{\gamma}{\gamma+1}\right)=\phi^{2}(1+\gamma)^{2} /\left(1+\gamma^{2}\right)$ and $\pi_{*}^{T}(r)<\pi_{*}^{I}(r)$ for some $r$. In this case, $\pi_{*}^{T}(r)>\pi_{*}^{I}(r)$ for all $r \in\left[0,2 \phi \frac{\gamma}{\gamma+1}\right]$. So $\pi_{*}^{T}(r)<\pi_{*}^{I}(r)$ for some $r>2 \phi \frac{\gamma}{\gamma+1}$. Moreover, since $\frac{1}{2} \phi(\gamma+1)>2 \phi$ (because $\gamma \geq 3$ ), we know that $\pi_{*}^{T}(r)>\pi_{*}^{I}(r)$ for all $r \in[2 \phi, \infty]$. It remains to analyze what happens for $r \in\left[2 \phi \frac{\gamma}{\gamma+1}, 2 \phi\right]$. We can check that $\pi_{*}^{I}(r)$ is concave in $\left[2 \phi \frac{\gamma}{\gamma+1}, 2 \phi\right]$. We can also check that $\pi_{*}^{T}(r)$ is convex in $\left[2 \phi \frac{\gamma}{\gamma+1}, \frac{1}{2} \phi(\gamma+1)\right]$ which means that $\pi_{*}^{T}(r)$ is convex in $\left[2 \phi \frac{\gamma}{\gamma+1}, 2 \phi\right]$ since $\frac{1}{2} \phi(\gamma+1)>2 \phi$ in this case $(\gamma \geq 3)$. From the fact that $\pi_{*}^{I}(r)$ is concave and $\pi_{*}^{T}(r)$ is convex in $\left[2 \phi \frac{\gamma}{\gamma+1}, 2 \phi\right]$, it follows that $\pi_{*}^{I}(r)$ and $\pi_{*}^{T}(r)$ cross two times. Let the crossing points be $r_{1}$ and $r_{2}$. If $\pi_{*}^{T}(2 \phi)>\lim _{r \uparrow 2 \phi} \pi_{*}^{I}(r)$, then $r_{2}<2 \phi$, else $r_{2}=2 \phi$ (strictly speaking it is not a crossing point in this case since $\pi_{*}^{I}$ is not continuous at $r=2 \phi$, but let us allow this abuse of language.) So in both cases, we obtain that $\pi_{*}^{T}(r)<\pi_{*}^{I}(r)$ if $r \in\left[r_{1}, r_{2}\right]$ and $\pi_{*}^{T}(r)>\pi_{*}^{I}(r)$ if otherwise, as claimed.

Case 1.3: $\gamma$ is such that $\pi_{*}^{T}\left(2 \phi \frac{\gamma}{\gamma+1}\right)<\pi_{*}^{I}\left(2 \phi \frac{\gamma}{\gamma+1}\right)=\phi^{2}(1+\gamma)^{2} /\left(1+\gamma^{2}\right)$. This immediately implies that $\pi_{*}^{T}$ and $\pi_{*}^{I}$ cross once in $\left[0,2 \phi \frac{\gamma}{\gamma+1}\right]$. Denote this crossing point by $r_{1}$. From the 
fact that $\pi_{*}^{I}(r)$ is concave and $\pi_{*}^{T}(r)$ is convex in $\left[2 \phi \frac{\gamma}{\gamma+1}, 2 \phi\right]$, it follows that $\pi_{*}^{I}(r)$ and $\pi_{*}^{T}(r)$ cross at most once in $\left[2 \phi \frac{\gamma}{\gamma+1}, 2 \phi\right]$. Denote this crossing point by $r_{2}$. If $\pi_{*}^{T}(2 \phi)>\lim _{r \uparrow 2 \phi} \pi_{*}^{I}(r)$, then $r_{2}<2 \phi$, else $r_{2}=2 \phi$ (strictly speaking it is not a crossing point in this case since $\pi_{*}^{I}$ is not continuous at $r=2 \phi$, but we admit the abuse of language for the sake of brevity.) So in both cases, we obtain that $\pi_{*}^{T}(r)<\pi_{*}^{I}(r)$ if $r \in\left[r_{1}, r_{2}\right]$ and $\pi_{*}^{T}(r)>\pi_{*}^{I}(r)$ if otherwise, as claimed.

Step 2b (Case 2: $\gamma<3$ ): In this case $\frac{1}{2} \phi(\gamma+1)<2 \phi$. Again, several subcases must be considered:

Case 2.1: $\gamma$ is high enough so that $\pi_{*}^{T}(r)>\pi_{*}^{I}(r)$. Clearly in this case, the functions never cross and team is always better.

Case 2.2: $\gamma$ is such that $\pi_{*}^{T}\left(\frac{1}{2} \phi(\gamma+1)\right)>\pi_{*}^{I}\left(\frac{1}{2} \phi(\gamma+1)\right)$ and $\pi_{*}^{T}(r)<\pi_{*}^{I}(r)$ for some $r$. Since $\pi_{*}^{I}(r)$ is constant for $r<2 \phi \frac{\gamma}{\gamma+1}$ and is concave in $\left[2 \phi \frac{\gamma}{\gamma+1}, 2 \phi\right]$ and $\pi_{*}^{T}(r)$ is convex in $\left[2 \phi \frac{\gamma}{\gamma+1}, \frac{1}{2} \phi(\gamma+1)\right]$, it follows that $\pi_{*}^{T}(r)$ and $\pi_{*}^{I}(r)$ cross twice in $\left[0, \frac{1}{2} \phi(\gamma+1)\right]$. Moreover, one can check that in this case $\partial \pi_{*}^{T}(r) / \partial r>\partial \pi_{*}^{I}(r) / \partial r$ for all $r \in\left[\frac{1}{2} \phi(\gamma+1), 2 \phi\right]$. This implies that $\pi_{*}^{T}(r)>\pi_{*}^{I}(r)$ for all $r \in\left[\frac{1}{2} \phi(\gamma+1), 2 \phi\right]$. Thus, there must exist $r_{1}$ and $r_{2}$ such that $\pi_{*}^{I}(r)>\pi_{*}^{T}(r)$ for all $r \in\left[\frac{1}{2} \phi(\gamma+1), 2 \phi\right]$ and $\pi_{*}^{I}(r)<\pi_{*}^{T}(r)$ for the other values of $r$.

Case 2.3: $\gamma$ is such that $\pi_{*}^{T}\left(\frac{1}{2} \phi(\gamma+1)\right)<\pi_{*}^{I}\left(\frac{1}{2} \phi(\gamma+1)\right)$. Since $(a) \pi_{*}^{I}$ is constant and $\pi_{*}^{T}$ is decreasing for $r \in\left[0,2 \phi \frac{\gamma}{\gamma+1}\right]$ and $(b) \pi_{*}^{I}$ is concave and $\pi_{*}^{T}$ is convex in $\left[2 \phi \frac{\gamma}{\gamma+1}, \frac{1}{2} \phi(\gamma+1)\right]$, it follows that $\pi_{*}^{I}$ and $\pi_{*}^{T}$ cross at most once in $\left[0, \frac{1}{2} \phi(\gamma+1)\right]$. Now, we can check that in this case $\pi_{*}^{T}-\pi_{*}^{I}$ is convex in $\left[\frac{1}{2} \phi(\gamma+1), 2 \phi\right]$, which implies that $\pi_{*}^{T}(r)$ and $\pi_{*}^{I}(r)$ cross at most once in $r \in\left[\frac{1}{2} \phi(\gamma+1), 2 \phi\right]$. Since $\pi_{*}^{T}(2 \phi)>\pi_{*}^{I}(2 \phi)$, we know they cross (eventually at $r=2 \phi$ ). Therefore, there must exist $r_{1}$ and $r_{2}$ such that $\pi_{*}^{I}(r)>\pi_{*}^{T}(r)$ for all $r \in\left[\frac{1}{2} \phi(\gamma+1), 2 \phi\right]$ and $\pi_{*}^{I}(r)<\pi_{*}^{T}(r)$ for the other values of $r$.

Proof of Proposition 4. The proof is given in the following steps.

Step 1. (comparative statics for $r_{1}$ ) Recall from the proof of Proposition 3 , that if $r_{1}<\phi(1+\gamma) / 2, r_{1}$ solves

$$
\phi^{2}(1+\gamma)^{2} /\left(1+\gamma^{2}\right)=\phi^{2}+r_{1} K\left(r_{1}\right) / \gamma^{2},
$$

whereas if $r_{1} \geq \phi(1+\gamma) / 2, r_{1}$ solves

$$
\phi^{2}+2 r_{1}(\phi \gamma-r) / \gamma^{2}=\left(\phi^{2}(1+\gamma)^{2}+4 \phi(1+\gamma) r-4 r^{2}\right) / 2\left(1+\gamma^{2}\right) .
$$

If $r_{1}<\phi(1+\gamma) / 2$, then $\hat{\pi}_{*}^{T}(\phi(1+\gamma) / 2)<\hat{\pi}_{*}^{I}(\phi(1+\gamma) / 2)$. This holds only if $\gamma<4$. 015 4. From equation (15) one obtains $r_{1}=2 \phi \times\left(\gamma^{2} /\left(\gamma^{2}+1\right)\right) \times((\sqrt{2}+1) /(3+2 \sqrt{2}-\gamma))$ (this is the only root of equation (15) in the relevant interval of values of $r$, i.e., in $[\phi \gamma /(\gamma+$ $1), \phi(\gamma+1) / 2])$. Now, from direct inspection of $r_{1}$, it follows that $r_{1}$ is an increasing function of $\gamma$ in the interval $(1,4.0154)$.

If $r_{1}>\phi(1+\gamma) / 2$, from equation (16) one obtains $r_{1}=\frac{1}{2}(\sqrt{2}-1) \phi \gamma(\gamma-1)$ (this is the only positive root of equation (16)). Now, $\partial r_{1} / \partial \gamma=\frac{1}{2}(\sqrt{2}-1) \phi(2 \gamma-1)>0$.

Step 2. (comparative statics for $r_{2}$ ) Recall from the proof of Proposition 3 , that $r_{2}$ solves

$$
\phi^{2}=\left(\phi^{2}(1+\gamma)^{2}+4 \phi(1+\gamma) r_{2}-4 r_{2}^{2}\right) / 2\left(1+\gamma^{2}\right) .
$$

The only positive root of equation (17) is $r_{2}=\frac{1}{2} \phi(1+\sqrt{\gamma})^{2}$. Thus, $\partial r_{2} / \partial \gamma=\frac{1}{2} \phi(1+1 / \sqrt{\gamma})>$ 0 .

Step 3. (Upper threshold for $\gamma$ ) Define $\bar{\gamma}$ as the value of $\gamma$ for which $r_{1}=\phi \bar{\gamma}$. Now, for all $r \neq r_{1}, \pi_{*}^{T}>\pi_{*}^{I}$. To see this, note the following: for $r>r_{1}, \pi_{*}^{T}>\pi_{*}^{I}$ as $\pi_{*}^{I}$ is decreasing in $r$ for $r \in(\phi(1+\bar{\gamma}) / 2, \phi(1+\bar{\gamma}))$, but, by definition of $r_{1}$, for all $r>r_{1}=\phi \bar{\gamma}$, 
$\pi_{*}^{T}(r)=\pi_{*}^{T}\left(r_{1}\right)=\pi_{*}^{I}\left(r_{1}\right)$ (note that this implies $\left.r_{1}=r_{2}=\phi \bar{\gamma}\right)$. Also, for $r<r_{1}, \pi_{*}^{T}>\pi_{*}^{I}$. The argument is as follows. Because $\pi_{*}^{T}(\phi \bar{\gamma})=\pi_{*}^{I}(\phi \bar{\gamma})$, and for all $r \in(\phi(1+\bar{\gamma}) / 2, \phi \bar{\gamma})$, $\pi_{*}^{T \prime}(r)<\pi_{*}^{I \prime}(r)$ (see step 1 of the previous proof), $\pi_{*}^{T}(\phi(1+\bar{\gamma}) / 2)>\pi_{*}^{I}(\phi(1+\bar{\gamma}) / 2)$. Now, for all $r<\phi(1+\bar{\gamma}) / 2, \pi_{*}^{T}(r)>\pi_{*}^{T}(\phi(1+\bar{\gamma}) / 2)>\pi_{*}^{I}(\phi(1+\bar{\gamma}) / 2)=\pi_{*}^{T}(r)$. Now, because $r_{1}>\phi(1+\gamma) / 2$, for $\gamma=\bar{\gamma}, r_{1}$ must solve (16). But such an $r_{1}$ is increasing in $\gamma$ (by step 2). So, for all $\gamma>\bar{\gamma}, r_{1}>\phi \bar{\gamma}$, which is inadmissible by definition of $r_{1}$. Thus, $\pi_{*}^{T}>\pi_{*}^{I}$ for all $r$.

Step 4. (Lower threshold for $\gamma$ ) Define $\underline{\gamma}$ as the value of $\gamma$ for which $r_{1}=\phi(1+\underline{\gamma}) / 2$, or $\underline{\gamma}=4.0154$. For $\gamma>\gamma$ (and $\gamma<\bar{\gamma}$ ), $r_{1}$ must solve equation (16) (see Step 1 of this proof). Now, for $\gamma \in(\underline{\gamma}, \bar{\gamma}), r_{1}$ is given by the solution to equation (16), and $r_{2}$ is given by the solution to equation (17). Therefore, $r_{2}-r_{1}=\frac{1}{2}(\sqrt{2}-1) \phi \gamma(\gamma-1)-\frac{1}{2} \phi(1+\sqrt{\gamma})^{2}$.

Step 5. Finally, compute $\partial\left(r_{2}-r_{1}\right) / \partial \gamma=\frac{1}{2}(1 / \sqrt{\gamma}+2 \gamma-\sqrt{2}(2 \gamma-1))$. Observe that $\partial\left(r_{2}-r_{1}\right) / \partial \gamma<0$ for $\gamma=\underline{\gamma}=4.0154$, and $\partial^{2}\left(r_{2}-r_{1}\right) / \partial \gamma^{2}<0$. Therefore, for all $\gamma>\underline{\gamma}$, $\partial\left(r_{2}-r_{1}\right) / \partial \gamma<0$.

Proof of Proposition 5. This proof closely follows the proof of Proposition 1. So, for the sake of brevity, we omit some of the details that are already discussed in the proof of Proposition 1 and highlight the key difference between this case and the proof of Proposition 1.

The key difference lies in the agents' $(I C)$ constraints. With substitutability between efforts, one obtains the following:

$$
\left\{\begin{array}{lll}
e_{1}=\frac{\beta_{A}(1-\theta \gamma)}{1-\theta^{2}}, & e_{2}=\frac{\beta_{A}(\gamma-\theta)}{1-\theta^{2}} & \text { if } \theta<1 / \gamma \\
e_{1}=0 & e_{2}=\gamma \beta_{A} & \text { if } \theta \geq 1 / \gamma
\end{array}\right.
$$

(the $I C_{B}^{I}$ is obtained from $I C_{A}^{I}$ by substituting $\beta_{A}$ by $\beta_{B}, e_{1}$ by $e_{3}$ and $e_{2}$ by $e_{4}$ ), and

$$
\left(I C_{A}^{T}\right) \quad\left\{\begin{array}{lll}
e_{1}=0, & e_{4}=\gamma \beta_{A 2} & \text { if } \beta_{A 1}<\gamma \theta \beta_{A 2} \\
e_{1}=\frac{\beta_{A 1}-\theta \gamma \beta_{A 2}}{1-\theta^{2}}, & e_{4}=\frac{\gamma \beta_{A 2}-\theta \beta_{A 1}}{1-\theta^{2}} & \text { if } \gamma \theta \beta_{A 2}<\beta_{A 1}<\frac{\gamma}{\theta} \beta_{A 2} \\
e_{1}=\beta_{A 1}, & e_{4}=0 & \text { if } \beta_{A 1}>\frac{\gamma}{\theta} \beta_{A 2}
\end{array}\right.
$$

(the $I C_{B}^{T}$ is obtained from $I C_{A}^{T}$ by substituting $\beta_{A 1}$ by $\beta_{B 2}, \beta_{A 2}$ by $\beta_{B 1}, e_{1}$ by $e_{3}$ and $e_{4}$ by $\left.e_{2}\right)$. Given this, by following the same steps as in Section 3, one obtains the firms' optimal profit functions.

Under individual accountability, the firm's optimal profit function is as follows. For $\theta<$ $1 / \gamma$

$$
\pi_{*}^{I}(r)=\left\{\begin{array}{ll}
\phi^{2} \xi(\gamma+1) \eta & \text { if } r \leq \phi \xi / 2 \\
4 r(\phi-r(\theta+1)+\gamma \phi) \eta & \text { if } \phi \xi / 2<r \leq \phi \xi \\
0 & \text { if } r>\phi \xi
\end{array},\right.
$$

where $\xi=(\gamma+1) /(\theta+1)$ and $\eta=(1-\theta) /\left(\gamma^{2}-2 \theta \gamma+1\right)$. For $\theta \geq 1 / \gamma$,

$$
\pi_{*}^{I}(r)=\left\{\begin{array}{ll}
\phi^{2} & \text { if } r \leq \gamma \phi / 2 \\
4 r(\gamma \phi-r) / \gamma^{2} & \text { if } \gamma \phi / 2<r \leq \gamma \phi \\
0 & \text { if } r>\gamma \phi
\end{array} .\right.
$$

In both cases, $\pi_{*}^{I}(r)$ is a continuous and decreasing function of $r$.

Under team accountability, the firm's optimal profit function is as follows. Let $\sigma=$ $\left(2 \theta \gamma+\gamma^{2}+1\right)(1-\theta) /(\theta+1)$. For $\sigma<\gamma^{2}$,

$$
\pi_{*}^{T}(r)=\left\{\begin{array}{ll}
2 \phi^{2} /(\theta+1) & \text { if } r \leq \gamma \phi /((\theta+1)(\gamma+1)) \\
r\left(\rho+\left(\rho^{2}+\vartheta\right)^{1 / 2}\right) / \gamma^{2} & \text { if } \gamma \phi /(\theta+1)(\gamma+1))<r \leq r_{0} \\
\phi^{2} & \text { if } r_{0}<r \leq \gamma \phi / 2 \\
4 r(\gamma \phi-r) / \gamma^{2} & \text { if } \gamma \phi / 2<r \leq \gamma \phi \\
0 & \text { if } r>\gamma \phi
\end{array},\right.
$$


where $\rho=2 \gamma \phi(\gamma+1)-2 r\left(2 \theta \gamma+\gamma^{2}+1\right), \vartheta=(2 \gamma \phi(\gamma-1))^{2}(1-\theta) /(\theta+1)$ and $r_{0}=$ $\gamma \phi /\left(2\left(\gamma+1-\sigma^{1 / 2}\right)\right)$. For $\sigma>\gamma^{2}$,

$$
\pi_{*}^{T}(r)= \begin{cases}2 \phi^{2} /(\theta+1) & \text { if } r \leq \gamma \phi /[(\theta+1)(\gamma+1)] \\ r\left(\rho+\left[\rho^{2}+\vartheta\right]^{1 / 2}\right) / \gamma^{2} & \text { if } \gamma \phi /[(\theta+1)(\gamma+1)]<r \leq r_{1} \\ 4 r(\gamma \phi-r) / \gamma^{2} & \text { if } r_{1}<r \leq \gamma \phi \\ 0 & \text { if } r>\gamma \phi\end{cases}
$$

where $r_{1}=\phi\left(\gamma+2 \theta \gamma+\gamma^{2}-\chi^{1 / 2}\right) /(2(2 \theta+\gamma))$ and $\chi=2 \gamma \theta(\theta+\gamma-1)\left(2 \theta \gamma+\gamma^{2}+1\right) /(1+\theta)$. In both cases, $\pi_{*}^{T}(r)$ is a continuous and decreasing function of $r$. The reminder of the proof is given in the following steps.

Step 1. We first show that for $\theta \geq 1 / \gamma, \pi_{*}^{I}(r) \leq \pi_{*}^{T}(r)$. Note that $\theta>1 / \gamma$ implies that $(1+\theta) /(1-\theta)>\left(2 \theta \gamma+\gamma^{2}+1\right) / \gamma^{2}$, which is equivalent to $\sigma<\gamma^{2}$. Thus one has to compare $\pi_{*}^{I}(r)$ as given in (18) with $\pi_{*}^{T}(r)$ as given in (19). For $r>\gamma \phi / 2, \pi_{*}^{I}(r)=\pi_{*}^{T}(r)$. For $r \leq \gamma \phi / 2, \pi_{*}^{T}(r) \geq \phi^{2}$, since $\pi_{*}^{T}(r)$ is decreasing in $r$ and $\pi_{*}^{T}(\gamma \phi / 2)=\phi^{2}$. Thus, for $r \leq \gamma \phi / 2, \pi_{*}^{T}(r) \geq \phi^{2}=\pi_{*}^{I}(r)$.

Step 2. We next show that for $\theta<1 / \gamma, \pi_{*}^{T}(r)$ and $\pi_{*}^{I}(r)$ must intersect at a unique point for $r \in[0, \max \{\gamma \phi, \phi \xi\}]$. First note that when $\theta<1 / \gamma, \pi_{*}^{T}(0)=2 \phi^{2} /(\theta+1)>$ $\phi^{2} \xi(\gamma+1) \eta=\pi_{*}^{I}(0)$. Also note that when $\theta<1 / \gamma, \gamma<\xi$, implying that $\gamma \phi<\phi \xi$. Thus, $\pi_{*}^{I}(\gamma \phi)>0=\pi_{*}^{T}(\gamma \phi)$. So, by Mean Value Theorem, there must exist a value of $r \in[0, \phi \xi]$, say $\widehat{r}$, such that $\pi_{*}^{T}(\widehat{r})=\pi_{*}^{I}(\widehat{r})$. Furthermore, independent of whether $\sigma<\gamma^{2}$ or $\sigma>\gamma^{2}$, when $\theta<1 / \gamma, \pi_{*}^{T}(\phi \xi / 2)<\pi_{*}^{I}(\phi \xi / 2)$. Hence, $\widehat{r} \in(0, \phi \xi / 2)$. In $(0, \phi \xi / 2), \pi_{*}^{I}(r)$ is constant, and $\pi_{*}^{T}(r)$ is either constant or decreasing. Moreover, $\forall r \in(0, \phi \xi / 2)$ such that $\partial \pi_{*}^{T}(r) / \partial r=0$, $\pi_{*}^{T}(r) \neq \pi_{*}^{T}(r)$. Thus, $\pi_{*}^{T}(r)$ and $\pi_{*}^{I}(r)$ intersect only once in $(0, \phi \xi / 2)$. Finally, note that $\left|\partial \pi_{*}^{T}(r) / \partial r\right| \geq\left|\partial \pi_{*}^{I}(r) / \partial r\right| \forall r \in(\phi \xi / 2, \phi \xi)$, implying that $\pi_{*}^{T}(r)$ and $\pi_{*}^{I}(r)$ do not intersect for $r \in(\phi \xi / 2, \phi \xi)$.

Proof of Proposition 6. Following steps analogous to derivation in the main model, one the derive the optimal contract and profit functions under individual and team accountability. Under individual accountability, the optimal contract and the associated profit function are:

$$
\beta_{*}^{I}(r)= \begin{cases}\phi\left(k_{1}+\gamma k_{2}\right) /\left(k_{1}+\gamma^{2} k_{2}\right) & \text { if } r \leq \frac{1}{2} \phi\left(k_{1}+\gamma k_{2}\right) \\ \left(2 \phi\left(k_{1}+\gamma k_{2}\right)-2 r\right) /\left(k_{1}+\gamma^{2} k_{2}\right) & \text { if } \frac{1}{2} \phi\left(k_{1}+\gamma k_{2}\right)<r<\phi\left(k_{1}+\gamma k_{2}\right) \\ 0 & \text { if } r \geq \phi\left(k_{1}+\gamma k_{2}\right)\end{cases}
$$

and

$$
\pi_{*}^{I}(r)=\left\{\begin{array}{ll}
J \phi^{2} \frac{1}{2} \frac{\left(k_{1}+\gamma k_{2}\right)^{2}}{\left(k_{1}+\gamma^{2} k_{2}\right)} & \text { if } \quad r \leq \frac{1}{2} \phi\left(k_{1}+\gamma k_{2}\right) \\
2 J \frac{\phi\left(k_{1}+\gamma k_{2}\right) r-r^{2}}{k_{1}+\gamma^{2} k_{2}} & \text { if } \quad \frac{1}{2} \phi\left(k_{1}+\gamma k_{2}\right)<r<\phi\left(k_{1}+\gamma k_{2}\right) \\
0 & \text { if } r \geq \phi\left(k_{1}+\gamma k_{2}\right)
\end{array} .\right.
$$

Under team accountability, the optimal contract and the associated profit function are:

$$
\begin{array}{lll}
\beta_{1}^{T^{*}}=0, & \beta_{2}^{T^{*}}=\frac{\beta}{k_{2}} & \text { if } \beta<\phi k_{2} \frac{\gamma-1}{\gamma^{2}} \\
\beta_{1}^{T^{*}}=\frac{\gamma^{2} \beta-\phi(\gamma-1) k_{2}}{k_{2}+\gamma^{2} k_{1}}, & \beta_{2}^{T^{*}}=\frac{\beta+\phi(\gamma-1) k_{1}}{k_{2}+\gamma^{2} k_{1}} & \text { if } \quad \phi k_{2} \frac{\gamma-1}{\gamma^{2}} \leq \beta<\phi \frac{\left(\gamma k_{1}+k_{2}\right)}{\gamma} \\
\beta_{1}^{T^{*}}=\phi, & \beta_{B 1}^{T^{*}}=\frac{\phi}{\gamma} & \text { if } \quad \beta \geq \phi \frac{\left(\gamma k_{1}+k_{2}\right)}{\gamma},
\end{array}
$$

and

$$
\pi_{*}^{T}(r)= \begin{cases}J \phi^{2} \frac{\left(k_{1}+k_{2}\right)}{2} & \text { if } r \leq \frac{1}{2} \gamma \phi \frac{k_{1}+k_{2}}{k_{2}+\gamma k_{1}} \\ J r K(r) / \gamma^{2} & \text { if } \frac{1}{2} \gamma \phi \frac{k_{1}+k_{2}}{k_{2}+\gamma k_{1}}<r \leq \frac{1}{2}(\gamma+1) \phi \\ J r \frac{2}{\gamma^{2}} k_{2}(\gamma \phi-r) & \text { if } \frac{1}{2}(\gamma+1) \phi<r<\phi \gamma \\ 0 & \text { if } r \geq \phi \gamma\end{cases}
$$


where $K(r)=A+\left[A^{2}+\gamma^{2}(1-\gamma)^{2} \phi^{2} k_{1} k_{2}\right]^{1 / 2}$ and $A=\phi \gamma\left(k_{2}+\gamma k_{1}\right)-r\left(k_{2}+\gamma^{2} k_{1}\right)$. Note that $\frac{1}{2} \phi\left(k_{1}+\gamma k_{2}\right)>\frac{1}{2} \phi(\gamma+1)$, since $k_{1}, k_{2} \geq 1$. The remainder of the proof is given by the following steps.

Step 1. We first show that for $r \in\left(\frac{1}{2} \gamma \phi\left(k_{1}+k_{2}\right) /\left(k_{2}+\gamma k_{1}\right), \phi \gamma\right],\left|\partial \pi_{*}^{I} / \partial r\right|<\left|\partial \pi_{*}^{T} / \partial r\right|$. For $r \in\left(\frac{1}{2} \gamma \phi\left(k_{1}+k_{2}\right) /\left(k_{2}+\gamma k_{1}\right), \phi\left(k_{1}+\gamma k_{2}\right) / 2\right]$ this is obvious, since $\partial \pi_{*}^{I} / \partial r=0$ and $\partial \pi_{*}^{T} / \partial r<0$. For $r \in\left[\phi\left(k_{1}+\gamma k_{2}\right) / 2, \phi \gamma\right], \partial \pi_{*}^{I} / \partial r=2 J\left(\phi k_{1}-2 r+\gamma \phi k_{2}\right) /\left(k_{1}+\gamma^{2} k_{2}\right)$ and $\partial \pi_{*}^{T} / \partial r=2 J k_{2}(\gamma \phi-2 r) / \gamma^{2}$. Since $\partial \pi_{*}^{I} / \partial r<0$ and $\partial \pi_{*}^{T} / \partial r<0,\left|\partial \pi_{*}^{I} / \partial r\right|<\left|\partial \pi_{*}^{T} / \partial r\right|$ is equivalent to $\partial \pi_{*}^{I} / \partial r-\partial \pi_{*}^{T} / \partial r>0$. This difference is increasing in $r$, since $\partial\left(\partial \pi_{*}^{I} / \partial r-\right.$ $\left.\partial \pi_{*}^{T} / \partial r\right) / \partial r=4 J\left(k_{1} k_{2}-\gamma^{2}+\gamma^{2} k_{2}^{2}\right) /\left(\left(k_{1}+\gamma^{2} k_{2}\right) \gamma^{2}\right)>0$ (recall that $\left.k_{2} \geq 1\right)$. Thus, it is sufficient to show that $\partial \pi_{*}^{I} / \partial r-\partial \pi_{*}^{T} / \partial r>0$ when $r=\phi\left(k_{1}+\gamma k_{2}\right) / 2$. This condition is equivalent to $2 \gamma^{-2}\left(k_{1}-\gamma+\gamma k_{2}\right) J \phi k_{2}>0$ (again, recall that $k_{2} \geq 1$ ).

Step 2. We now show $\pi_{*}^{I}$ and $\pi_{*}^{T}$ cross only once. Because $\pi_{*}^{T}\left(\frac{1}{2} \gamma \phi\left(k_{1}+k_{2}\right) /\left(k_{2}+\gamma k_{1}\right)\right)$ $=J \phi^{2}\left(k_{1}+k_{2}\right) / 2>J \phi^{2} \frac{1}{2}\left(k_{1}+\gamma k_{2}\right)^{2} /\left(k_{1}+\gamma^{2} k_{2}\right)=\pi_{*}^{I}\left(\frac{1}{2} \gamma \phi\left(k_{1}+k_{2}\right) /\left(k_{2}+\gamma k_{1}\right)\right)$ and $\pi_{*}^{T}(\phi \gamma)=$ $0<\pi_{*}^{I}(\phi \gamma)$, by Mean Value Theorem, there must exist a value of $r \in\left[\frac{1}{2} \gamma \phi\left(k_{1}+k_{2}\right) /\left(k_{2}+\right.\right.$ $\left.\left.\gamma k_{1}\right), \phi \gamma\right]$, say $r^{* *}$, such that $\pi_{*}^{T}\left(r^{* *}\right)=\pi_{*}^{I}\left(r^{* *}\right)$. Moreover, since $\left|\partial \pi_{*}^{I} / \partial r\right|<\left|\partial \pi_{*}^{T} / \partial r\right|$ for $r \in\left(\frac{1}{2} \gamma \phi\left(k_{1}+k_{2}\right) /\left(k_{2}+\gamma k_{1}\right), \phi \gamma\right], r^{* *}$ must be unique.

\section{REFERENCES}

[1] Baker, G., R. Gibbons, and K. Murphy. "Subjective Performance Measures in Optimal Incentive Contracts." Quarterly Journal of Economics, Vol. 109 (1994), pp. 1125-56.

[2] Banker, R., and S. Datar. "Sensitivity, Precision and Linear Aggregation of Signals for Performance Evaluation." Journal of Accounting Research Vol. 27 (1989), pp. 21-39.

[3] Bar-Issac, H. "Something to Prove: Reputation in Teams." RAND Journal of Economics, Vol. 38 (2007), pp. $495-511$.

[4] Bartol, K. and L. Hagmann. "Team-Based Pay Plans: A Key to Effective Teamwork." Compensation and Benefits Review, Vol. 24 (1992), pp. 24-29.

[5] Bernheim, D., and M. Whinston. "Multimarket Contact and Collusive Behavior." RAND Journal of Economics, Vol. 21 (1990), pp. 1-26.

[6] Besanko, D., Regibeau, P., and K. Rockett. "A Multi-task Principal-Agent Approach to Organizational Form." Journal of Industrial Economics, Vol. 53 (2005), pp. 437-467.

[7] Banker, R., and S. Datar. "Sensitivity, Precision, and Linear Aggregation of Signals for Performance Evaluation." Journal of Accounting Research, Vol. 27 (1989), pp. 21-39.

[8] Bull, C. "The Existence of Self-Enforcing Implicit Contracts." Quarterly Journal of Economics, Vol. 102 (1987), pp. 147-60.

[9] Che, Y., and S. Yoo. "Optimal Incentives for Teams." The American Economic Review, Vol. 91 (2001), pp. 525-541.

[10] Chen, J., H. Hong, M. Huang, and J. Kubik. "Does Fund Size Erode Mutual Fund Performance? The Role of Liquidity and Organization." American Economic Review, Vol. 94, (2004), pp. 1276-1302.

[11] Corts, K. "Teams versus Individual Accountability: Solving Multi-Task Problems Through Job Design." RAND Journal of Economics, Vol. 38 (2007), pp. 467-479.

[12] Dewatripont, M., I. Jewitt, and J. Tirole. "Multitask Agency Problems: Focus and Task Clustering." European Economic Review, Vol. 44 (2000), pp. 869-877.

[13] Fudenberg, D., D. Levine, and E. Maskin. "The Folk Theorem with Imperfect Public Information." Econometrica, Vol. 62 (1994), pp. 997-1039.

[14] Getmansky, M., Lo, A. and S. Wei. "Sifting through the Wreckage: Lessons from Recent Hedge Fund Liquidations." Journal of Investment Management, Vol. 2 (2004), pp. 6-38.

[15] Gibbons, R. and K. Murphy. "Optimal Incentive Contracts in the Presence of Career Concerns: Theory and Evidence." Journal of Political Economy, Vol. 100 (1992), pp. 468-505.

[16] Holmström, B. "Moral Hazard in Teams." Bell Journal of Economics, Vol. 13 (1982), pp. 324-340.

[17]__ _ and P. Milgrom. "Multi-task Principal Agent Problems: Incentive Contracts, Asset Ownership and Job Design." Journal of Law, Economics and Organsation, Vol. 7 (1991), pp. 24-52.

[18] Itoh, H. "Incentives to Help in Multiagent Situations." Econometrica, Vol. 59 (1991), pp. 611-637.

[19] Inderst, R., and M. Ottaviani. "(Mis)selling Through Agents." Working paper, London School of Economics, 2008. 
[20] Kvaloy, O., and T. Olsen. "Team Incentives in Relational Employment Contracts." Journal of Labor Economics, Vol. 24 (2006), pp. 139-169.

[21] Levin, J. "Relational Incentive Contracts." American Economic Review, Vol. 93 (2003), pp. 835-847.

[22] __ "Multilateral Contracting and the Employment Relationship." Quarterly Journal of Economics, Vol. 117 (2002), pp. 1075-1103.

[23] MacLeod, B., and J. Malcomson. "Implicit Contracts, Incentive Compatibility, and Involuntary Unemployment." Econometrica, Vol. 57 (1989), pp. 447-80.

[24] Massa, M., J. Reuter, and E. Zitzewitz. "When Should Firms Share Credit with Employees? Evidence from Anonymously Managed Mutual Funds." Mimeo, Darthmouth College, 2008.

[25] Prather, L., and K. Middleton. "Are $N+1$ Heads Better than One? The Case of Mutual Fund Managers." Journal of Economic Behavior and Organization, Vol. 47 (2002), pp. 103-120.

[26] Ramakrishnan, R., and A. Thakor. "Cooperation Versus Competition in Agency." Journal of Law, Economics, $\&$ Organization, Vol. 7 (1991), pp. 248-283.

[27] Rayo, L. "Relational Incentives and Moral Hazard in Teams." Review of Economic Studies, Vol. 74 (2007), pp. 937-963.

[28] Schöttner, A. "Relational Contracts, Multitasking, and Job Design." Journal of Law, Economics, and Organization, Vol. 24 (2008), pp. 138-162.

[29] Shaw, D. and C. Schneier. "Team Measurement and Rewards: How Some Companies Are Getting It Right." Human Resource Planning, Vol. 18 (1995), pp. 34-49.

[30] Wilder, J. "Contingency Fees and Incentives in Commercial Lines Insurance." Ph.D. Thesis, Massachusetts Institute of Technology, Department of Economics, (2002). 